\title{
North Atlantic Simulations with the Hybrid Coordinate Ocean Model (HYCOM): Impact of the Vertical Coordinate Choice, Reference Pressure, and Thermobaricity
}

\author{
Eric P. Chassignet, Linda T. Smith, and George R. Halliwell \\ Rosenstiel School of Marine and Atmospheric Science, University of Miami, Miami, Florida \\ RAINER BLECK \\ Atmospheric and Climate Science, Los Alamos National Laboratory, Los Alamos, New Mexico
}

(Manuscript received 2 December 2002, in final form 28 May 2003)

\begin{abstract}
The viability of a generalized (Hybrid) Coordinate Ocean Model (HYCOM), together with the importance of thermobaricity and the choice of reference pressure, is demonstrated by analyzing simulations carried out using the World Ocean Circulation Experiment (WOCE) Community Modeling Experiment (CME) Atlantic basin configuration. The standard hybrid vertical coordinate configuration is designed to remain isopycnic throughout as much of the water column as possible while smoothly making a transition to level (pressure) coordinates in regions with weak vertical density gradients, such as the surface mixed layer, and to terrain-following coordinates in shallow-water regions. Single-coordinate (pressure or density) experiments illustrate the flexibility of the model but also bring forward some of the limitations associated with such a choice. Hybrid experiments with potential density referenced to the surface $\left(\sigma_{\theta}\right)$ and to $20 \mathrm{MPa}(\sim 2000 \mathrm{~m})\left(\sigma_{2}\right)$ illustrate the increased influence of pressure errors with increasing distance from the reference pressure. The $\sigma_{\theta}$ hybrid experiment does not properly reproduce the northward flow of Antarctic Bottom Water (AABW), and large errors in near-surface pressure gradients in the $\sigma_{2}$ experiment produce a wind-driven gyre circulation that is too strong, when compared with observations, and a North Atlantic Current that follows an unrealistic path. These near-surface and nearbottom pressure errors are removed when thermobaric effects are included, resulting in a more accurate representation of the upper-ocean gyre circulation, the northward AABW flow near the bottom, and the meridional overturning circulation and heat flux.
\end{abstract}

\section{Introduction}

The numerical modeling studies carried out for the North and equatorial Atlantic basin over the past several decades have demonstrated advances both in model architecture and in the availability of computational resources for the scientific community. Perhaps the most noticeable aspect of this progression has been the evolution from simulations on coarse-resolution horizontal/ vertical grids outlining basins of simplified geometry and bathymetry and forced by idealized stresses, to fineresolution simulations incorporating realistic coastal definition and bottom topography, forced by observational data on relatively short timescales (Smith et al. 2000b; Chassignet and Garraffo 2001).

A remaining issue of importance for the design of numerical models is that of the choice of vertical coordinate. Recent model comparison exercises performed in Europe [Dynamics of North Atlantic Models (DYNAMO); Willebrand et al. 2001] and in the United States [Data Assimilation and Model Evaluation Experiment (DAMÉE); Chassignet et al. 2000] have shown that no single vertical coordinate-depth, density, or the

Corresponding author address: Dr. Eric P. Chassignet, RSMAS/ MPO, University of Miami, 4600 Rickenbacker Causeway, Miami, FL 33149-1098.

E-mail: echassignet@rsmas.miami.edu terrain-following sigma — can be by itself optimal everywhere in the ocean. These and earlier comparison studies (Chassignet et al. 1996, hereinafter CSBB; Roberts et al. 1996, Marsh et al. 1996) have shown that the models considered are able to simulate the large-scale characteristics of the North Atlantic circulation reasonably well but that the interior water mass distribution and associated thermohaline circulation are strongly influenced by localized processes that are not represented equally by each model's vertical discretization. Ideally, a numerical ocean model should retain its water mass characteristics for centuries (a characteristic of isopycnic coordinates), have high vertical resolution in the surface mixed layer and maintain sufficient vertical resolution in unstratified or weakly stratified regions of the ocean (a characteristic of depth coordinates), and have high vertical resolution in coastal regions (a characteristic of terrain-following coordinates).

The realization that there is no optimal vertical coordinate has led to the development of a generalizedcoordinate ocean model, the Hybrid Coordinate Ocean Model (HYCOM) (Bleck 2002; see online at http:// hycom.rsmas.miami.edu). The implementation of the generalized coordinate in HYCOM follows the theoretical foundation set forth in Bleck and Boudra (1981) and Bleck and Benjamin (1993); that is, each coordinate surface is assigned a reference isopycnal. The model 
continually checks whether grid points lie on their reference isopycnals and, if not, attempts to move them vertically toward the reference position. However, the grid points are not allowed to migrate when this would lead to excessive crowding of coordinate surfaces. Thus, vertical grid points can be geometrically constrained to remain at a fixed depth while being allowed to join and follow their reference isopycnals in adjacent areas (Bleck 2002). The default configuration in HYCOM is one that is isopycnal in the open stratified ocean but smoothly reverts to a terrain-following coordinate in shallow coastal regions and to fixed pressure-level coordinates (hereinafter referred to as $p$ ) in the surface mixed layer and/or unstratified seas. In doing so, the model combines the advantages of the different types of coordinates in optimally simulating coastal and openocean circulation features. It is left to the user to define the coordinate separation constraints that control regional transitions among the three coordinate choices.

HYCOM is a generalized-coordinate mass-conserving ocean numerical model and can therefore be configured for and integrated with only one coordinate, that is, isopycnic, pressure-level, or terrain-following. Some single-coordinate experiments will be discussed later on, not only to illustrate the flexibility of the model but also to bring forward some of the limitations associated with such a choice.

The first goal of this paper is to demonstrate the viability of the generalized (hybrid) coordinate ocean model by validating it against observations and previous simulations configured for the North and equatorial Atlantic. Building on past studies with the Community Modeling Experiment (CME) configuration (Holland and Bryan 1994; CSBB; Smith et al. 2000a, hereinafter $\mathrm{SCB}$ ), we take advantage of the built-in vertical coordinate flexibility of the hybrid model in order to examine the effect of the coordinate choice(s) on the model's ability to accurately represent the water mass distribution and three-dimensional circulation of the Atlantic. The second goal is to document the importance of thermobaricity (i.e., the modulation of seawater compressibility by potential temperature anomalies) and the choice of reference pressure. Specifically, we address an issue that is present in models such as the Miami Isopycnic Coordinate Ocean Model (MICOM) or HYCOM that employ potential density either as the sole vertical coordinate or as one component of the hybrid framework, namely, the question of how best to infer local buoyancy contrasts from a given potential density distribution based on a uniform reference pressure.

Experiments with potential density referenced to the surface $\left(\sigma_{\theta}\right)$ and to $20 \mathrm{MPa}(\sim 2000 \mathrm{~m})\left(\sigma_{2}\right)$ illustrate the increased influence of pressure errors with increasing distance from the reference pressure. The $\sigma_{\theta}$ hybrid experiment does not properly reproduce the northward flow of Antarctic Bottom Water (AABW), while large errors in near-surface pressure gradients in the $\sigma_{2}$ experiment produce a wind-driven gyre circulation that is too strong, when compared with observations, and a North Atlantic Current that follows an unrealistic path. These near-surface and near-bottom pressure errors are removed when thermobaric effects are included.

The paper is organized as follows: Section 2 describes the generalized-coordinate (hybrid) model and the configuration of the experiments. Section 3 first compares a HYCOM integration with a Kraus-Turner mixed layer with simulations performed with MICOM (the basis of the hybrid code). We then introduce the baseline HYCOM simulation with the $K$-profile parameterization (KPP) vertical mixing model and compare the results with simulations performed in pure isopycnic and pressure-level modes. We continue through a series of HY$\mathrm{COM}$ experiments that vary the choice of reference pressure for the vertical coordinate, focusing on differences in the surface circulation, the representation of water masses, the overturning circulation and meridional heat transport, and the interior deep circulation. Section 4 summarizes and discusses the results.

\section{The numerical model and its configuration}

The generalized-coordinate HYCOM used in this study retains many of the characteristics of MICOM while allowing coordinate surfaces to locally deviate from isopycnals wherever the latter may fold, outcrop, or generally provide inadequate vertical resolution in portions of the model domain. The freedom to adjust the vertical spacing of the coordinate surfaces in HYCOM simplifies the numerical implementation of several physical processes (mixed layer detrainment, convective adjustment, sea ice modeling, ....) without robbing the model of the basic and numerically efficient layer architecture that is characteristic of MICOM throughout most of the ocean's volume. Documentation of the hybrid-model numerics, including details of the grid generator that allows coordinate layers to maintain finite thickness (therefore becoming nonisopycnic), can be found in Bleck (2002) and online at http://hycom. rsmas.miami.edu.

Several vertical mixing parameterizations have been implemented in HYCOM. The model can be integrated with a Kraus-Turner (K-T; Kraus and Turner 1967) slab mixed layer, a KPP (Large et al. 1994, 1997) nonslab vertical mixing formulation, a Price-Weller-Pinkel (PWP; Price et al. 1986) slab mixed layer, a MellorYamada level-2.5 turbulence closure model (Mellor and Yamada 1982), or a Goddard Institute for Space Studies (GISS) level-2.0 turbulence closure model (Canuto et al. 2001, 2002). A detailed discussion of the relative performance of these five mixed layers can be found in Halliwell (2003). In this paper, we will first use the slab Kraus-Turner mixed layer for comparison with earlier MICOM experiments and then the KPP vertical mixing scheme (widely used in large-scale ocean simulations) for the remaining experiments.

The semi-implicit KPP closure scheme is easily im- 
TABLE 1. Summary of the model experiments.

\begin{tabular}{|c|c|c|c|}
\hline Expt & Vertical mixing parameterization & Vertical coordinate & Remarks \\
\hline EXP-M' & Kraus-Turner & $\sigma_{\theta}$ & Friedrich-Levitus equation of state \\
\hline EXP-M & Kraus-Turner & $\sigma_{\theta}$ & \\
\hline EXP-H ${ }_{\mathrm{KT}}$ & Kraus-Turner & Hybrid $\sigma_{\theta} / p$ & \\
\hline EXP-H & KPP & Hybrid $\sigma_{\theta} / p$ & \\
\hline EXP-H & KPP & $\sigma_{\theta}$ & See text for details \\
\hline $\mathrm{EXP}-\mathrm{H}_{\mathrm{P}}$ & KPP & $p$ & $\begin{array}{l}\sigma_{\theta} \text { initial conditions; no diffusive tensor } \\
\text { rotation; no GM90* parameterization }\end{array}$ \\
\hline $\mathrm{EXP}-\mathrm{H} \sigma_{2}$ & KPP & Hybrid $\sigma_{2} / p$ & \\
\hline $\mathrm{EXP}-\mathrm{H} \sigma_{2}^{*}$ & KPP & Hybrid $\sigma_{2} / p$ & With correction for thermobaricity \\
\hline
\end{tabular}

*GM90: Gent and McWilliams (1990).

plemented in HYCOM because of the model's ability to assign several coordinate surfaces to the oceanic mixed layer. In the KPP scheme, the surface boundary layer diffusivity and viscosity profiles are smoothly matched to the relatively weak diapycnal diffusivity and viscosity profiles of the interior ocean, to provide mixing throughout the water column. The contribution of background internal wave breaking, shear instability mixing, and double diffusion are parameterized for the ocean interior, while for the surface boundary layer, the influences of wind-driven mixing, surface buoyancy fluxes, and convective instability are accounted for (Large et al. 1994, 1997).

The computational domain for these experiments is identical to that in CSBB and SCB, namely, the domain of the World Ocean Circulation Experiment (WOCE) Community Modeling Experiment (CME) (Bryan and Holland 1989; U.S. WOCE 1993). The model basin comprises the North and equatorial Atlantic Ocean from $15^{\circ} \mathrm{S}$ to $65^{\circ} \mathrm{N}$, including the Caribbean Sea and Gulf of Mexico but excluding the Mediterranean Sea. Bottom topography is derived from a digital terrain dataset with $5^{\prime}$ resolution in latitude and longitude (ETOPO5). All surface boundary conditions are based on seasonal climatological datasets. The wind stress and wind work values are taken from the Hellerman and Rosenstein (1983) climatology. The surface thermal boundary conditions are specified by a linear bulk formula described by Han (1984), while those for freshwater flux are implemented in traditional fashion as a linear relaxation of the surface salinity toward the Levitus (1982) climatology. Open ocean boundaries are treated as closed but are outfitted with buffer zones in which the temperature $T$ and salinity $S$ are linearly relaxed toward their seasonally varying climatological values. These buffer zones restore the $T$ and $S$ fields to the Levitus climatology in order to approximately recover the vertical shear of the currents through geostrophic adjustment. The four buffer zones are located at the northern boundary and at the southern boundary ( $4^{\circ}$ latitude band), in the northwestern corner (representing the Labrador Sea), and in the Gulf of Cádiz (representing the Mediterranean Sea). The restoring timescale for the boundaries varies from 25 days at the inner edge linearly decreasing to 5 days at the walls. The time- scale for the Labrador Sea region is 25 days and is $1 \mathrm{yr}$ for the Mediterranean. For more details, the reader is referred to Bryan and Holland (1989) and Holland and Bryan (1994).

The horizontal grid is on a Mercator projection with resolution of $0.9^{\circ}$ longitude. The coefficients for the along-coordinate mixing of momentum and diffusivity of tracers are $1 \times 10^{4}$ and $2 \times 10^{3} \mathrm{~m}^{2} \mathrm{~s}^{-1}$, respectively. The coefficient for vertical mixing of tracers is $3 \times 10^{-5}$ $\mathrm{m}^{2} \mathrm{~s}^{-1}$, and the coefficient for smoothing of the isopycnals (equivalent to Gent and McWilliams 1990) is $1 \times 10^{3} \mathrm{~m}^{2} \mathrm{~s}^{-1}$. The quadratic bottom drag coefficient is $1.3 \times 10^{-3}$.

The present experiments (summarized in Table 1) are as follows,

- EXP-M' ${ }^{\prime}$ and EXP-M: MICOM with slab K-T mixed layer, $\sigma_{\theta}$ coordinate,

- EXP-H $\mathrm{H}_{\mathrm{KT}}$ : HYCOM with slab K-T mixed layer, hybrid $\sigma_{\theta} / p$ coordinate,

- EXP-H: HYCOM with KPP vertical mixing, hybrid $\sigma_{\theta} / p$ coordinate,

- EXP-H $\mathrm{H}_{\mathrm{ISO}}$ : HYCOM with KPP vertical mixing, predominantly $\sigma_{\theta}$ coordinate,

- EXP-H $\mathrm{H}_{\mathrm{P}}$ : HYCOM with KPP vertical mixing, pressure-level coordinate only, with $\sigma_{\theta}$ initial conditions,

- EXP-H $\sigma_{2}: \mathrm{HYCOM}$ with KPP vertical mixing, hybrid $\sigma_{2} / p$ coordinate, and

- EXP-H $\sigma_{2}^{*}$ : HYCOM with KPP vertical mixing, hybrid $\sigma_{2} / p$ coordinate with correction for thermobaricity.

The MICOM EXP-M' was integrated using the invertible Friedrich and Levitus (1972) equation of state with reference pressure at the surface, for comparison with the experiments of CSBB and SCB. The equation of state used for the remaining MICOM and HYCOM experiments is that of Brydon et al. (1999), with reference pressure at the surface for EXP-M, EXP- $\mathrm{H}_{\mathrm{KT}}$, EXP- $\mathrm{H}_{\mathrm{ISO}}, \mathrm{EXP}-\mathrm{H}_{\mathrm{P}}$, and EXP-H ( $\sigma_{\theta}$ coordinate), and at $20 \mathrm{MPa}(\sim 2000 \mathrm{~m})$ for EXP-H $\sigma_{2}$ and EXP-H $\sigma_{2}^{*}\left(\sigma_{2}\right.$ coordinate). Brydon et al. (1999) generated an invertible polynomial in potential temperature $\theta$, salinity $\mathrm{S}$, and pressure $p$, representing a best fit to the Jacket and McDougall (1995) UNESCO equation of state.

In all experiments but one $\left[\mathrm{EXP}-\mathrm{H}_{\mathrm{P}}\right.$, which has 30 
TABLE 2. Layer densities for EXP-M', M, $\mathrm{H}_{\mathrm{KT}}, \mathrm{H}\left(\sigma_{\theta}\right)$, EXP-H ISO $\left(\sigma_{\theta}\right)$, and EXP-H $\sigma_{2}, \mathrm{H} \sigma_{2}^{*}\left(\sigma_{2}\right)$.

\begin{tabular}{cccc}
\hline \hline & EXP-M $, \mathrm{M}, \mathrm{H}_{\mathrm{KT}}, \mathrm{H}$ & $\mathrm{EXP}-\mathrm{H}_{\mathrm{ISO}}$ & $\mathrm{EXP}-\mathrm{H} \sigma_{2}, \mathrm{H} \sigma_{2}^{*}$ \\
$\sigma_{\theta}$ & 23.50 & 29.04 \\
\hline 1 & 21.40 & 24.00 & 29.47 \\
2 & 21.80 & 24.25 & 29.89 \\
3 & 22.20 & 24.50 & 30.32 \\
4 & 22.60 & 24.70 & 30.80 \\
5 & 23.05 & 25.00 & 31.34 \\
6 & 23.55 & 25.28 & 31.82 \\
7 & 24.02 & 25.50 & 33.19 \\
8 & 24.70 & 25.70 & 34.23 \\
9 & 25.28 & 26.00 & 35.01 \\
10 & 25.77 & 26.18 & 35.59 \\
11 & 26.18 & 26.52 & 35.98 \\
12 & 26.52 & 26.80 & 36.27 \\
13 & 26.80 & 27.03 & 36.49 \\
14 & 27.03 & 27.22 & 36.66 \\
15 & 27.22 & 27.38 & 36.79 \\
16 & 27.38 & 27.52 & 36.89 \\
17 & 27.52 & 27.64 & 36.98 \\
18 & 27.64 & 27.74 & 37.04 \\
19 & 27.74 & 27.82 & 37.08 \\
20 & 27.82 & 27.88 & 37.11 \\
21 & 27.88 & 27.92 & 37.14 \\
22 & 27.92 & & \\
\hline
\end{tabular}

fixed levels as in the Geophysical Fluid Dynamics Laboratory (GFDL)-CME model (Bryan and Holland 1989)], there are 22 coordinate surfaces in the vertical with $\sigma_{\theta}$ and $\sigma_{2}$ values as listed in Table 2. The heaviest $16 \sigma_{\theta}$ coordinate densities are identical to those used in the 16-layer MICOM experiments of CSBB and SCB. In the $\sigma_{2}$ experiments, the densities were chosen so that the basinwide-averaged layer thicknesses for the initial state of the experiments, derived from the January climatology of Levitus (1982), would closely match those for the $\sigma_{\theta}$ experiments in the approximate depth range from 400 to $2000 \mathrm{~m}$. Above $400 \mathrm{~m}$, the $\sigma_{2}$ densities were chosen so as to create three fewer layers in that depth range than exist in the $\sigma_{\theta}$ case, making it possible to increase by three the number of $\sigma_{2}$ layers below 2000 $\mathrm{m}$. These additional deep layers permit resolution of the densest water masses (in particular, the Antarctic Bottom Water) that are not present in the $\sigma_{\theta}$ discretization because of the folding of $\sigma_{\theta}$ isopycnals in the AABW density range.

In all hybrid cases $\left(\sigma_{\theta}\right.$ and $\left.\sigma_{2}\right), 6$ layers are added above the 16 heaviest layers with densities outside the range of those found in the North and equatorial Atlantic. This ensures the existence of nonisopycnal coordinate surfaces (minimum separation of $10 \mathrm{~m}$ ) that will be maintained in a finite-thickness state by HYCOM's grid generator (Bleck 2002).

EXP- $\mathrm{H}_{\text {ISO }}$ differs from the other experiments in the sense that the 22 layers are active isopycnals, with the 6 lightest layers added to increase the vertical discretization near the surface above $\sigma_{\theta}=25.0$ (see Table 2 for details). The minimum distance between two isopycnals in EXP- $\mathrm{H}_{\mathrm{ISO}}$ is required to be $1 \mathrm{~m}$, in order for the tridiagonal matrix solver in the KPP vertical mixing scheme to accurately estimate the $K$ profile. This configuration is therefore isopycnic over all but a few meters of the entire water column.

Models such as HYCOM that are formulated in terms of potential density treat seawater as incompressible in the dynamic equations, ignoring in situ density variations along potential isopycnals caused by the thermobaric effect, that is, the modulation of seawater compressibility by potential temperature anomalies. Such variations can give rise to pressure forces, which, though weak, cannot be ignored in a general circulation model. Sun et al. (1999) have introduced a modified potential density, termed the "virtual potential density," to be used as a basis for dynamic height computations in the dynamic equations of the model, accounting for the difference between buoyancy and potential density. Transformation from Cartesian $(x, y, z)$ to potential density $\left(x, y, \alpha_{r}\right)$ coordinates under hydrostatic conditions changes the pressure gradient term in the horizontal momentum equation into

$$
-\alpha \boldsymbol{\nabla}_{z} p=-\boldsymbol{\nabla}_{\alpha_{r}} M+p \boldsymbol{\nabla}_{\alpha_{r}} \alpha,
$$

where $M=p \alpha+g z$ is the Montgomery potential and $\alpha$ is specific volume. When the in situ specific volume is used, the two terms on the right side of (1) are much larger than their residual, thus introducing a large truncation error into a numerical model. The virtual potential density, however, matches the in situ density in its ability to capture the ocean dynamics without introduction of the truncation error [see Sun et al. (1999) for details and the appendix for a summary]. EXP-H $\sigma_{2}^{*}$ is identical to EXP- $\mathrm{H} \sigma_{2}$ except for incorporation of the virtual potential density (hereinafter denoted $\sigma_{2}^{*}$ ) that takes into account the buoyancy anomalies caused by thermobaricity.

\section{The experiments}

In this section, we document the differences in model response to the choices of vertical discretization and reference pressure. A discussion is provided in section 4. All experiments (listed in section 2) are initialized with temperature and salinity for January conditions from the Levitus (1982) climatology and then integrated for 20 years. Unless otherwise noted, the results presented in this section are for the average of the final 3 years of integration.

\section{a. Comparison to earlier studies: $E X P-M^{\prime}$ and $E X P-M$}

Our reference set of experiments is as described in the comparison exercise of CSBB between the MICOM (version 2.6) and GFDL models. The purpose of this section is to briefly document some of the differences that are seen between the earlier MICOM simulations and those performed with the latest version of the code (version 2.8).

In addition to improvements in the numerics, MICOM version 2.8 employs a different equation of state from 

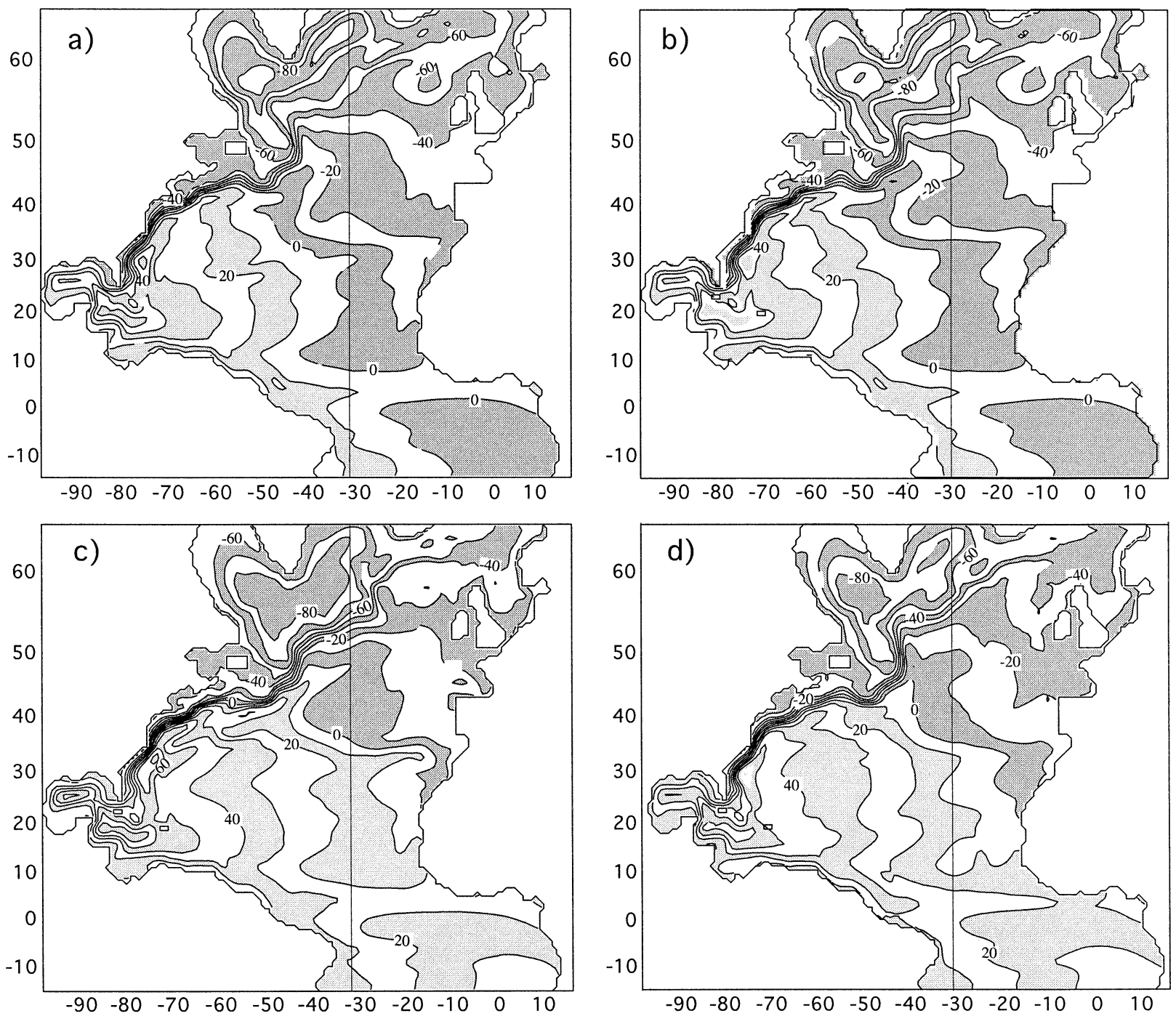

FIG. 1. Sea surface height $(\mathrm{cm})$, mean years 18-20. Contour interval is $10 \mathrm{~cm}$. (a) EXP-M', (b) EXP-M, (c) EXP-H $\mathrm{H}_{\mathrm{KT}}$, and (d) EXP-H. Vertical line is along $31.8^{\circ} \mathrm{W}$.

that used in version 2.6. Therefore, in order to assess the impact of the version-2.8 numerics on the solution, EXP-M' was integrated with the version-2.8 code and the Friedrich and Levitus (1972) equation of state used in 2.6. The differences between the EXP-M' $\mathrm{M}^{\prime}$ solution and the version-2.6 solution (EXP-MW; CSBB) are relatively small, with a maximum heat transport in EXP$\mathrm{M}^{\prime}$ of $1.0 \mathrm{PW}$, versus 1.15 PW in EXP-MW, and a maximum overturning in EXP-M' ${ }^{\prime}$ of $17 \mathrm{~Sv}$, versus 19 $\mathrm{Sv}$ in EXP-MW $\left(\mathrm{Sv} \equiv 10^{6} \mathrm{~m}^{3} \mathrm{~s}^{-1}\right)$. The largest difference is seen in the 3-yr average March mixed layer depth. EXP-MW has two distinct areas of deep convection in the Labrador and Irminger Basins with an average March mixed layer depth of $3000 \mathrm{~m}$ (Fig. 10a in SCB). The March mixed layer depths in EXP-M' (not illustrated), on the other hand, are shallower in both the Labrador and Irminger Basins, with an average of 900 $\mathrm{m}$. Deepening of the mixed layer in winter depends on preconditioning, which is determined by lateral advection of temperature and salinity. Since the forcing is the same in the two experiments, we surmise that the differences in mixed layer depth result from the time evolution of the temperature and salinity profiles, which differs in the two experiments because of changes in the mixed layer detrainment scheme and to a lesser extent numerical improvements in the tracer advection routine.

EXP-M differs from EXP-M' only in the equation of state used. The Brydon et al. (1999) polynomial expression allows for an invertible equation of state in $\theta$, $S$, and $p$ [Friedrich and Levitus (1972) used the in situ temperature $T$ ]. The differences after 20 years (Figs. 1a, b) are small and are, for the most part, due to differences in the initial conditions (e.g., the initial interface depths, Fig. 2) that arise from the use of in situ versus potential temperature. 

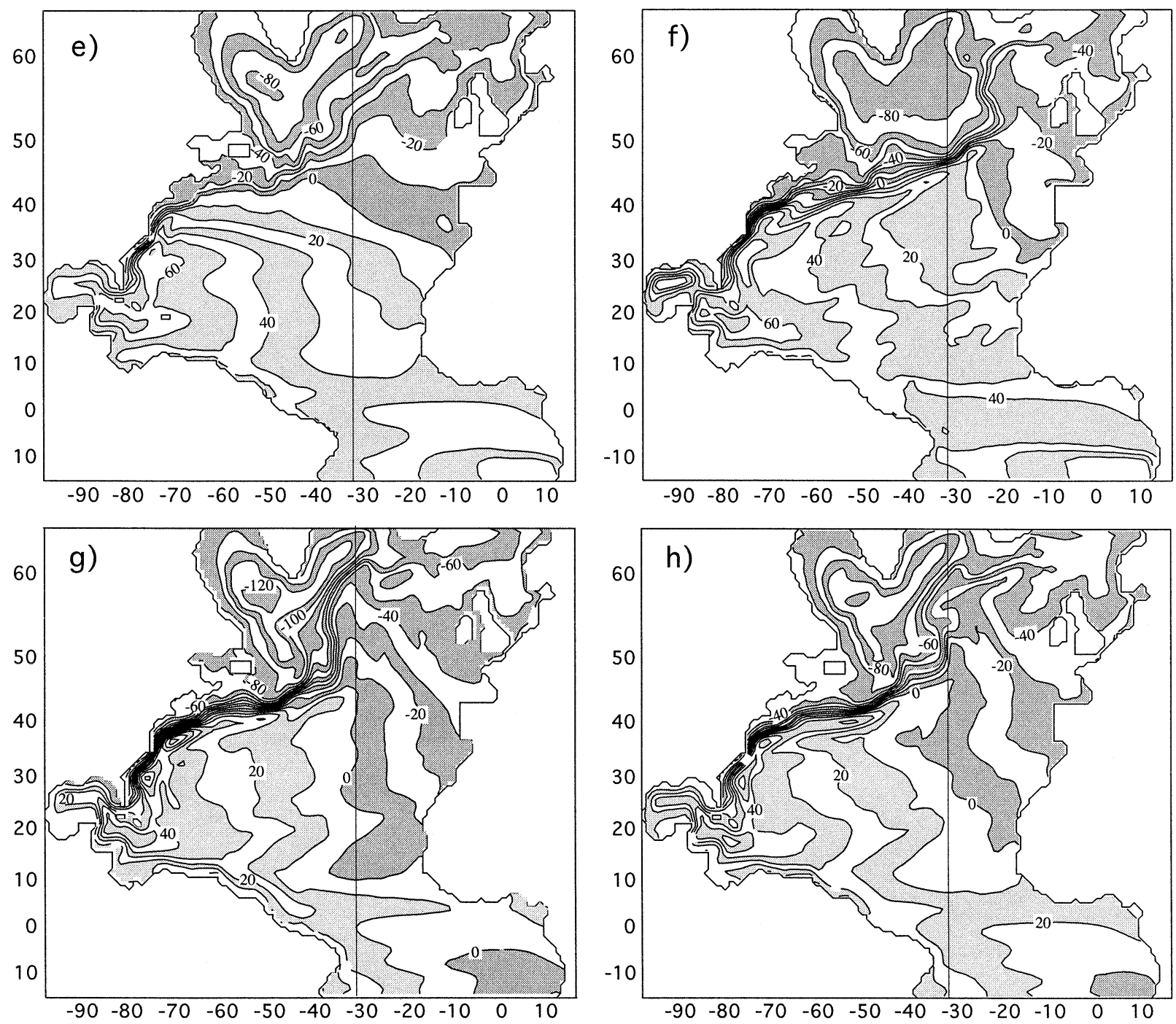

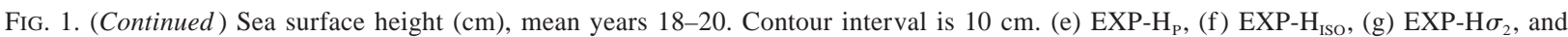
(h) EXP-H $\sigma_{2}^{*}$. Vertical line is along $31.8^{\circ} \mathrm{W}$.

\section{b. Surface circulation, as represented by the sea surface height (SSH)}

Figure 1 displays the mean SSH for eight experiments $\left(\mathrm{M}^{\prime}, \mathrm{M}, \mathrm{H}_{\mathrm{KT}}, \mathrm{H}, \mathrm{H}_{\mathrm{P}}, \mathrm{H}_{\mathrm{ISO}}, \mathrm{H} \sigma_{2}\right.$, and $\left.\mathrm{H} \sigma_{2}^{*}\right)$. The $\mathrm{SSH}$ is used here as a proxy for the surface circulation. The discussion will primarily focus on two aspects of the circulation, the strength/path of the western boundary current in the subtropical gyre and the strength/extent of the subpolar gyre.

The strength of the surface circulation, as measured by the difference between the subtropical SSH maximum and subpolar SSH minimum, is of the same order of magnitude in all experiments except EXP-H $\sigma_{2}$ (Fig. $1 \mathrm{~g}$ ), which is substantially stronger. Of the seven experiments with similar circulation strength, EXP- $\mathrm{H}_{\mathrm{P}}$ (Fig. 1e), with fixed coordinate levels, develops a much more diffuse flow between the gyres after the Gulf
Stream separates from the western boundary. The SSH field of EXP- $\mathrm{H}_{\mathrm{P}}$ is more diffuse and exhibits weaker gradients across the basin than seen in its hybrid and isopycnic counterparts (see SSH profile along $65^{\circ} \mathrm{W}$ in Fig. 3). The $\sigma_{2}$-coordinate EXP-H $\sigma_{2}$ (Fig. 1 g), on the other hand, exhibits a much stronger SSH gradient across both lobes of the subpolar gyre, across the North Atlantic Current (NAC) along the southern and eastern edges of the subpolar gyre, and across the Gulf Stream along $65^{\circ} \mathrm{W}$ (Fig. 3). The stronger surface circulation in EXP-H $\sigma_{2}$ is the result of inaccurate pressure gradients at the surface, because the reference pressure in that experiment is at $20 \mathrm{MPa}(\sim 2000 \mathrm{~m})$ and the density variations along potential isopycnals caused by thermobaricity are not taken into account. The use of the virtual potential density, $\sigma_{2}^{*}$, in EXP-H $\sigma_{2}^{*}$ (Fig. 1h) corrects for those variations, implying that the surface pres- 

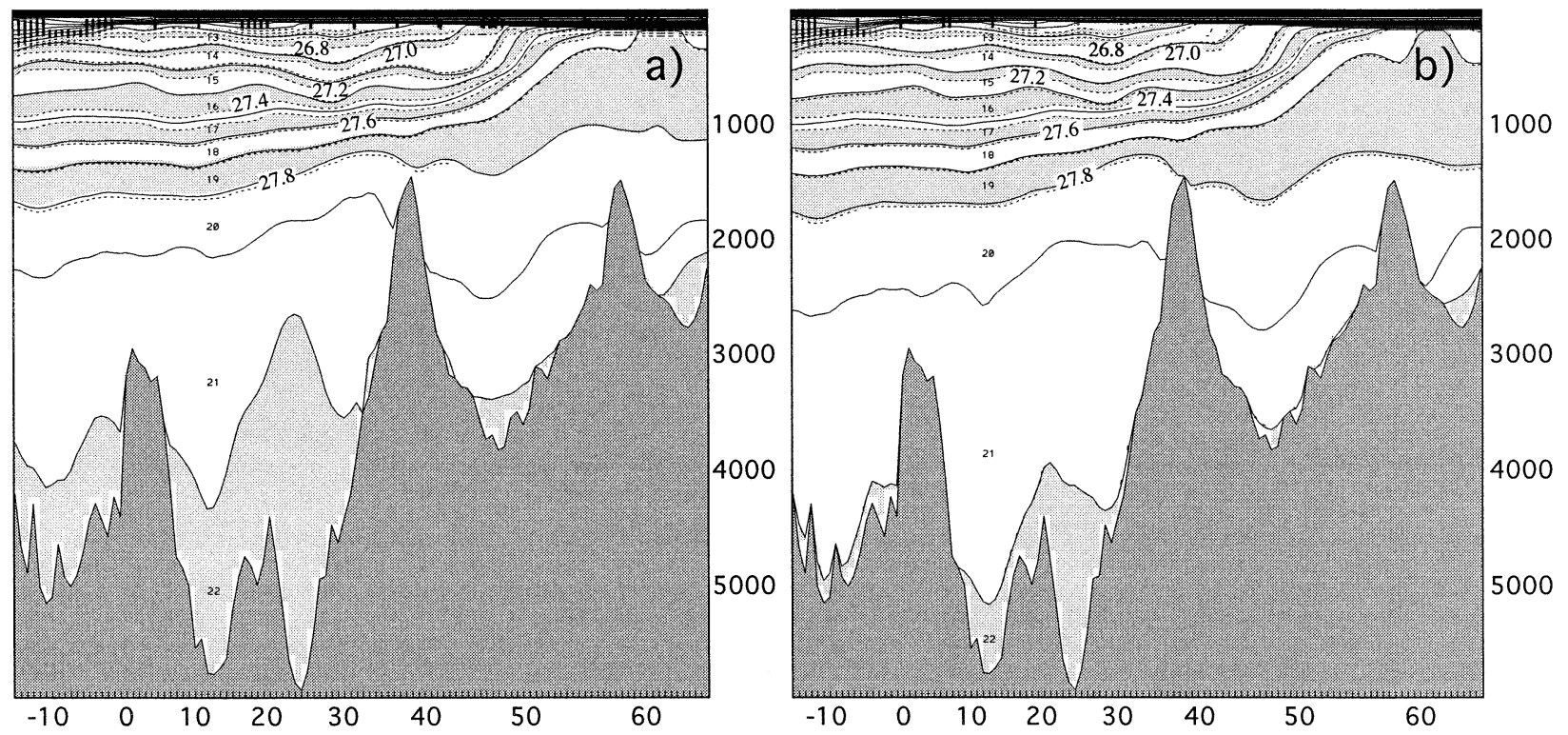

FIG. 2. Initial conditions along $31.8^{\circ} \mathrm{W}$ : (a) EXP-M' and (b) EXP-M. Contour interval is 0.1 for density $\left(\sigma_{\theta}\right)$. Solid lines are layer interfaces. Integers are layer numbers. Depths are shown in meters on vertical axis.

sure gradients in EXP-H $\sigma_{2}^{*}$ should theoretically be in close agreement with those of the $\sigma_{\theta}$ experiments $\mathrm{M}^{\prime}$, $\mathrm{M}, \mathrm{H}_{\mathrm{KT}}$, and $\mathrm{H}$ (Figs. 1a-d), which have a reference pressure at the surface. The SSH gradient in EXP$\mathrm{H} \sigma_{2}^{*}$ across the Gulf Stream along $65^{\circ} \mathrm{W}$ (Fig. 3) is indeed similar to that of the $\sigma_{\theta}$ experiments. The large differences in surface circulation between EXP-H $\sigma_{2}$ and EXP-H $\sigma_{2}^{*}$ (Figs. 1g,h) illustrate the importance of the

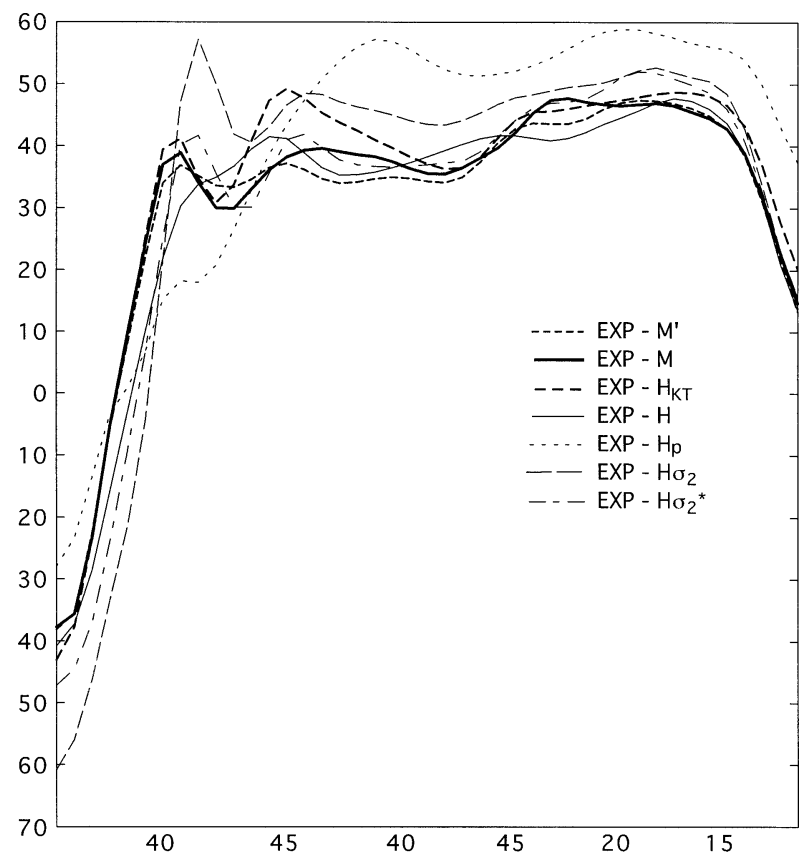

FIG. 3. Sea surface height along $65^{\circ} \mathrm{W}(\mathrm{cm})$, mean years $18-20$. Latitude is shown on horizontal axis. choice of reference pressure and of the proper representation of thermobaric effects.

The other notable difference among the experiments illustrated by Fig. 1 is in the path of the NAC. The path can vary significantly from one experiment to the other and is, to a large extent, dependent upon the strength of the subpolar gyre. Experiments with weaker subpolar gyres (EXP-M', M, $\mathrm{H}_{\mathrm{KT}}, \mathrm{H}, \mathrm{H}_{\mathrm{P}}$; Figs. 1a-e) have a NAC that turns north at Flemish Cap at $45^{\circ} \mathrm{W}$ before turning east at between $50^{\circ}$ and $55^{\circ} \mathrm{N}$. The eastward turn is more sharply defined in EXP-M', $M$, and $H$ than in EXP- $\mathrm{H}_{\mathrm{KT}}$ and $\mathrm{H}_{\mathrm{P}}$, and is thus in better agreement with observations (Dietrich et al. 1980; Lazier and Wright 1993). Both experiments with $20-\mathrm{MPa}$ reference pressure (EXP-H $\sigma_{2}$ and EXP-H $\sigma_{2}^{*}$, Figs. $1 \mathrm{~g}, \mathrm{~h}$ ) exhibit a stronger subpolar gyre than the experiments with surface reference pressure. Without a proper representation of the thermobaric effects $\left(\mathrm{EXP}-\mathrm{H} \sigma_{2}\right)$, the subpolar gyre is intensified and the NAC extends farther east $\left(35^{\circ} \mathrm{W}\right)$ before turning north. With thermobaricity (EXP-H $\sigma_{2}^{*}$ ), the strength of the subpolar gyre is more in line with that of experiments $\mathrm{M}^{\prime}, \mathrm{M}, \mathrm{H}_{\mathrm{KT}}, \mathrm{H}$, and $\mathrm{H}_{\mathrm{P}}$, and the NAC branches out northward and northeastward at Flemish Cap $\left(45^{\circ} \mathrm{W}\right)$, although the northeastward turn occurs farther to the south than in the other experiments.

Proper model representation of the subpolar gyre strength and of the NAC pathway is the result of a fine balance between mixed layer response and preconditioning of the deep water masses. For example, a small change in model formulation, namely, the use of biharmonic instead of harmonic (Laplacian) viscosity, led to a shutdown of convective events in the Labrador Sea in the German Family of Linked Atlantic Model Experiments (FLAME; C. Böning 2002, personal com- 


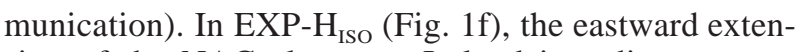
sion of the NAC almost to Ireland is a direct consequence of the poor representation of mixed layer processes in the subpolar gyre. Only three of the EXP- $\mathrm{H}_{\text {ISo }}$ isopycnic layers are active in that region (not illustrated), which is an insufficient number for the KPP closure to be properly implemented (Large et al. 1997; Halliwell 2003). In an additional experiment (not illustrated), 14 isopycnal layers were added in $\mathrm{EXP}_{-} \mathrm{H}_{\mathrm{ISO}}$ (for a total of 36 layers) in order to increase the vertical discretization of the subpolar gyre. This allowed a proper representation of the mixed layer physics in the subpolar region and hence a good representation of the NAC pathway, but at a greater computational cost than that of the hybrid approach.

\section{c. Mixed layer depth and ventilation patterns}

\section{1) MIXED LAYER DEPTH}

Halliwell (2003) provides an extensive review of the performance of the mixed layer parameterizations available for use in HYCOM. In this section, we investigate the model mixed layer's sensitivity to the vertical coordinate choice. The 3-yr average March mixed layer thicknesses are displayed in Fig. 4 for experiments $M$, $\mathrm{H}_{\mathrm{KT}}, \mathrm{H}, \mathrm{H}_{\mathrm{P}}, \mathrm{H} \sigma_{2}$, and $\mathrm{H} \sigma_{2}^{*}$. While the large-scale patterns are qualitatively similar among all experiments, the mixed layer is seen to be deepest over the northern subtropical gyre in the MICOM EXP-M (Fig. 4a), which uses the MICOM 2.8 bulk K-T mixed layer. The HY-

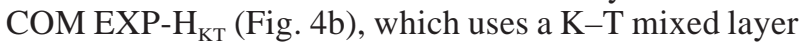
model designed for hybrid coordinates, also produces a thicker mixed layer over the same region in comparison to the four experiments that use KPP mixing. However, the HYCOM EXP- $\mathrm{H}_{\mathrm{KT}}$ mixed layer is not as thick as the MICOM EXP-M mixed layer, indicating that the Kraus-Turner model performs differently between the isopycnic and hybrid coordinate implementations. Halliwell (2003) observed similar behavior in low-resolution Atlantic simulations, and attributes this difference to issues related to the numerical implementation of the isopycnic and hybrid versions since the models employ the same TKE balance to govern entrainment and detrainment. As documented in Bleck et al. (1989, 1992), a rather complex mixed layer detrainment algorithm is required in MICOM because of its discrete density structure. The detrained water must have a density that matches the density of the isopycnic layer receiving the water. This is achieved by "unmixing" the fossil mixed layer into two sublayers with the lower one having the required density to be detrained and the upper one matching the temperature of the actual mixed layer. This requirement causes the EXP-M mixed layer spring retreat to be slower than observed and slower than in EXP$\mathrm{H}_{\mathrm{KT}}$, which does not require a special detrainment algorithm. The numerical difficulties in implementing the $\mathrm{K}-\mathrm{T}$ mixed layer in hybrid coordinates arise because the mixed layer base is no longer coincident with a model vertical coordinate, requiring special bookkeeping to estimate property jumps across the base (Halliwell 2003). The tendency for Kraus-Turner physics to produce a winter mixed layer across the northern subtropical gyre that is too deep is still evident in EXP-H $\mathrm{H}_{\mathrm{KT}}$ despite the changes in numerical implementation.

Large differences in maximum winter mixed layer depth are also evident over the subpolar gyre among the remaining experiments (Fig. 4). Mixed layer response varies among the experiments since its deepening in winter depends on preconditioning, which is determined by lateral advection of temperature and salinity. In weakly stratified regions, large changes in mixed layer depths can result from small changes in temperature and salinity that arise from the different vertical discretizations (isopycnic, pressure level, hybrid) as well as the different reference pressure choices. According to observations, outside of the northern buffer zone (i.e., south of $61^{\circ} \mathrm{N}$ ) there are two areas where deep convection occurs: in the Labrador Sea and in the southwest Irminger Sea (Pickart et al. 2003). The maximum mixed layer depth in the Labrador Sea varies from 700-800 $\mathrm{m}$ in the Kraus-Turner EXP-M and EXP-H $\mathrm{KT}_{\mathrm{KT}}$ (Figs. $4 \mathrm{a}, \mathrm{b})$ to $1600-2000 \mathrm{~m}$ in the KPP cases EXP-H, $\mathrm{H}_{\mathrm{P}}$, $\mathrm{H} \sigma_{2}$, and $\mathrm{H} \sigma_{2}^{*}$ (Figs. 4c-f). The distribution of the deepest part of the mixed layer in the Labrador region also differs among the experiments, being more localized in the $\sigma_{\theta}$ experiments than in those with $\sigma_{2}$ coordinate. In the latter (EXP-H $\sigma_{2}$ and EXP-H $\sigma_{2}^{*}$; Figs. 4e,f), deep convection also occurs within the Labrador Current. With one exception (EXP-H $\mathrm{KT}_{\mathrm{KT}}$; Fig. 4b), all experiments also exhibit deepening of the mixed layer in a broad area to the east and southeast of Greenland. Within that region, the deepest convection sites (on the order of $1000 \mathrm{~m}$ ) are found in the Irminger Sea only in the two $\sigma_{2}$ experiments EXP-H $\sigma_{2}$ and EXP-H $\sigma_{2}^{*}$ (Figs. 4e,f). The mixed layer deepening east of Greenland in EXP$\mathrm{H}_{\mathrm{KT}}$ (Fig. 4b) is localized in a region farther offshore than in the remaining experiments.

\section{2) Ventilation PATterns of THE SUbTROPiCAL GYRE}

The ventilation of the subtropical gyre is governed by the existence of areas of strong subduction rates along the southern flank of the deep winter mixing region, where the southward Sverdrup flow carries water away from the base of the winter mixed layer. The subtropical gyre primarily ventilates on density surfaces between $\sigma_{\theta}=26.5$ and 27.2 (New et al. 2001). In this section, we investigate the ventilation pathways in the model subtropical gyre on two surfaces, $\sigma_{\theta}=26.52$ and 27.03, in two experiments, EXP-H and EXP-H $\sigma_{2}^{*}$. Following New et al. (2001), potential vorticity is defined as $q=\rho_{0}^{-1} f \partial \rho / \partial z$, where $\rho$ is the fluid density with a reference value $\rho_{0}$ (here taken as $1026 \mathrm{~kg} \mathrm{~m}^{-3}$ ) and $f$ is the Coriolis parameter. Away from the boundary cur- 

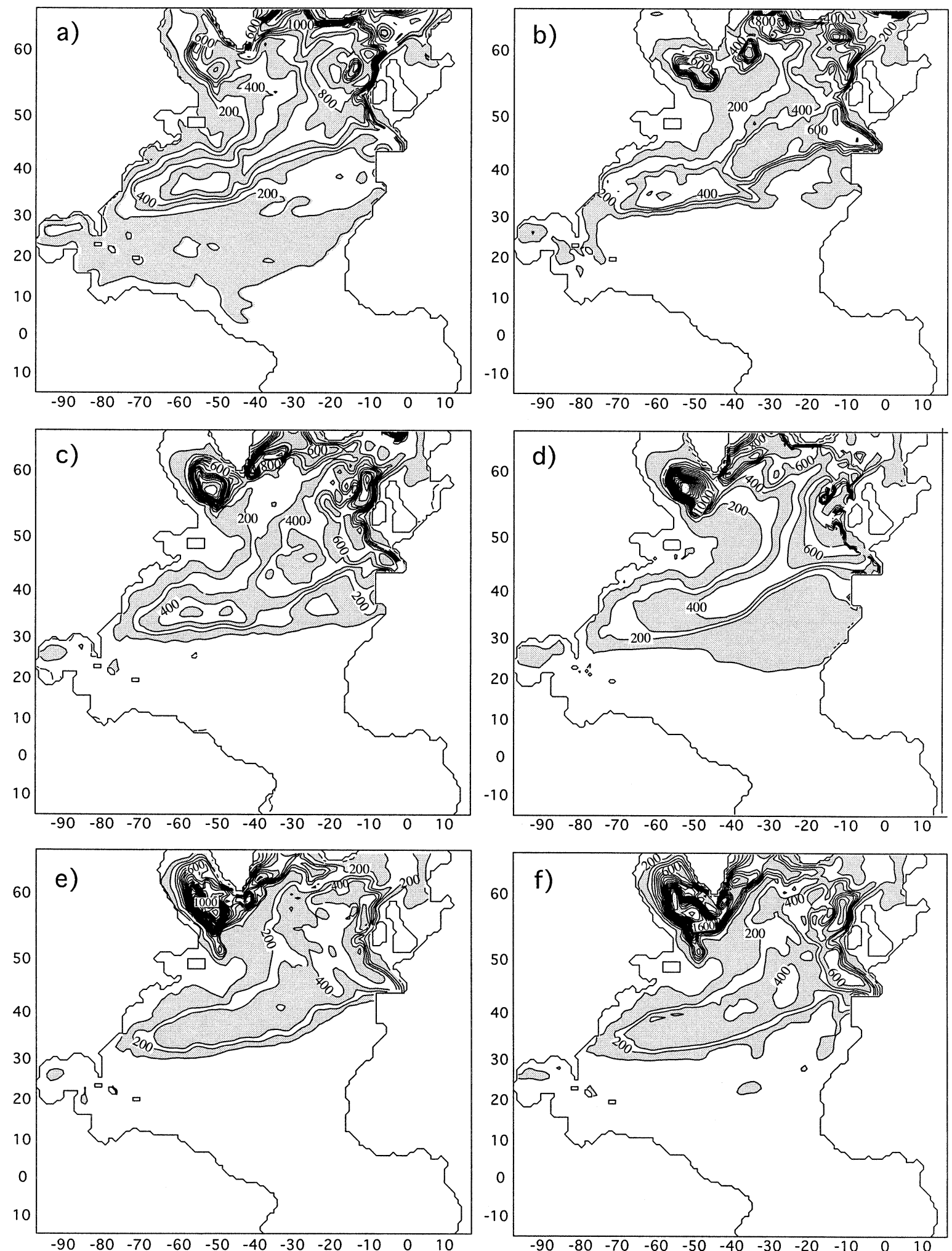

FIG. 4. Mixed layer depth (m), Mar mean years 18-20. Contour interval is $100 \mathrm{~m}$. (a) EXP-M, (b) EXP-H ${ }_{\mathrm{KT}}$, (c) EXP-H, (d) EXP-H EXP-H $\sigma_{2}$, and (f) EXP-H $\sigma_{2}^{*}$. 

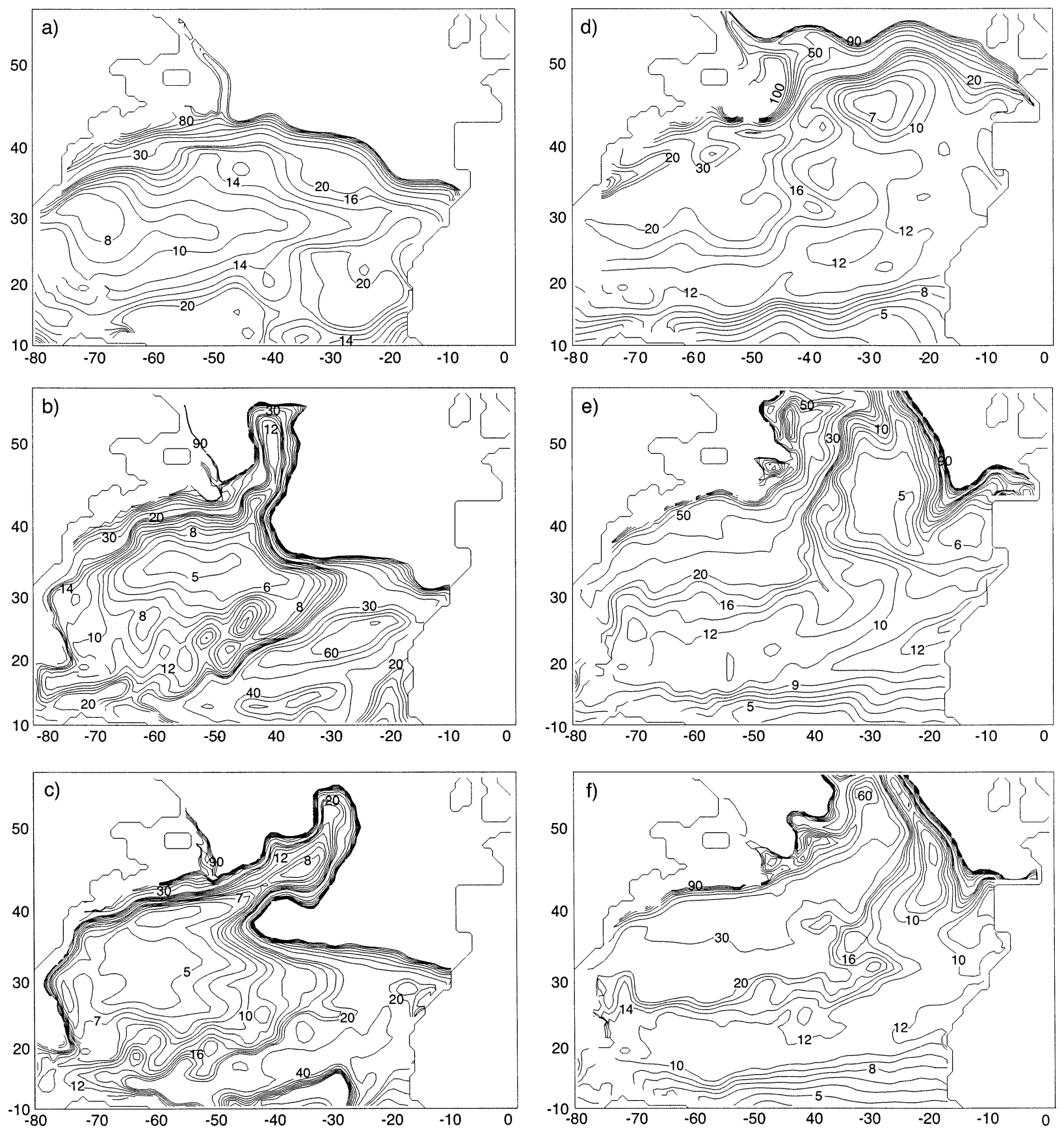

FIG. 5. Potential vorticity $\left(q \times 10^{11} \mathrm{~m}^{-1} \mathrm{~s}^{-1}\right)$ for (left) $\sigma_{\theta}=26.52$ and (right) $\sigma_{\theta}=27.03$. (a),(d) Initial conditions (Levitus); (b),(e) EXP$\mathrm{H}$ (Mar mean years 18-20); (c),(f) EXP-H $\sigma_{2}^{*}$ (Mar mean years 18-20). Contour interval is $1.0\left(q \times 10^{11} \leq 10\right), 2.0\left(10<q \times 10^{11} \leq 20\right)$, $10\left(q \times 10^{11}>20\right)$

rent systems, relative vorticity can be neglected in comparison with $f$, and the modeled $q$ can then be easily compared with climatological datasets. The $q$ fields are presented for the month of March when layers outcrop at the base of the mixed layer and when subduction is most active. Comparisons are made with the Levitus (1982) climatology, which was used for the model initial conditions, in order to assess the changes induced by the model circulation.

On the $26.52 \sigma_{\theta}$ surface, the mode water is observed to exist primarily in the Sargasso Sea (Fig. 5a) with $q$ values of less than 10. In both EXP-H and EXP-H $\sigma_{2}^{*}$ (Figs. 5b,c), the principal location of the mode water is farther north than in the climatology because of the 
northward path of the modeled Gulf Stream, which results in a more northerly outcrop of the interior layers. The minimum $q$ values in both cases are smaller than in the climatology (Fig. 5a) and cover a larger area, thus implying that more 26.52 mode water is present in the numerical simulations than in the climatology. The location of the lowest $q$ value is in better agreement with the climatology in EXP-H $\sigma_{2}^{*}$ than in EXP-H. However, the low potential vorticity on the 26.52 surface in EXP$\mathrm{H}$ extends farther to the east than in EXP-H $\sigma_{2}^{*}$. In the DYNAMO comparison exercise (New et al. 2001), this eastward advection appeared only in model experiments that produced strong Azores Currents. The fact that EXP-H $\sigma_{2}^{*}$ has a weaker Azores Current than EXP-H (Figs. 1d,h), and no eastward extension of the low potential vorticity on the 26.52 surface, supports that conclusion. New et al. (2001) also surmised that a stronger Azores Current will also tend to block the southward escape of heavier mode water south of $35^{\circ} \mathrm{N}$. Potential vorticity diagrams on the $27.03 \sigma_{\theta}$ surface (Figs. 5d,e,f) do indeed show that the heavier mode water is confined to the north of the Azores Current in EXP-H (Fig. 5e) and that the mode water can escape southward near the eastern boundary in EXP-H $\sigma_{2}^{*}$ (Fig. 5f).

Both EXP-H and EXP-H $\sigma_{2}^{*}$ include relaxation to the Levitus (1982) climatological $T$ and $S$ in the Gulf of Cádiz, which effectively acts as a source region for the Azores Current (Jia 2000; Özgökmen et al. 2001). Examination of the salinity fields in these experiments and in the climatology (not shown) reveals that the tongue of high salinity extending westward from the Gulf at $\sim 1000 \mathrm{~m}$ depth in EXP-H $\sigma_{2}^{*}$ more closely resembles that seen in the climatology than does the corresponding feature in EXP-H. This implies that the vertical velocities associated with the relaxation are smaller in EXP$\mathrm{H} \sigma_{2}^{*}$ than in EXP-H, resulting in a weaker Azores current signature in the former (Fig. 1h) than in the latter (Fig. 1d). The similarity between the salinity feature in the climatology and that in the $\sigma_{2}^{*}$ experiment could be a consequence of a better representation of the pressure gradients and circulation in the Gulf of Cádiz in EXP$\mathrm{H} \sigma_{2}^{*}$ (and therefore less erosion of the salinity maximum) than in EXP-H.

\section{d. Cross sections}

\section{1) $\operatorname{AT} 31.8^{\circ} \mathrm{W}$}

In this subsection, we discuss the interior water mass distribution at the end of the 20-yr integration by examining meridional cross sections at $31.8^{\circ} \mathrm{W}$ for four experiments (EXP-H, EXP-H $\mathrm{P}_{\mathrm{P}}, \mathrm{EXP}-\mathrm{H} \sigma_{2}$, and EXP$\mathrm{H} \sigma_{2}^{*}$; Fig. 6). By comparing EXP-H and EXP-H $\mathrm{P}_{\mathrm{P}}$, we can assess the impact of choosing hybrid versus fixed coordinates and, by comparing EXP-H, EXP-H $\sigma_{2}$, and EXP-H $\sigma_{2}^{*}$, we can assess the impact of the reference pressure choice and the correction for thermobaricity.

Figure 6 illustrates two of the main differences be- tween the fixed coordinate EXP-H $\mathrm{H}_{\mathrm{P}}$ (Fig. 6b) and the hybrid experiment EXP-H (Fig. 6a): (i) most of the dense water belonging to layer $22\left(\sigma_{\theta} \simeq 27.92\right)$ has disappeared in $\mathrm{EXP}-\mathrm{H}_{\mathrm{P}}$ after 20 years (see Fig. 10 in section $3 \mathrm{~d}$ for a discussion of its time evolution), and (ii) density gradients are weaker in EXP-H $\mathrm{P}_{\mathrm{P}}$ than in EXP-H. The divergence in deep-water mass structures over time between model results in level and isopycnic coordinates, first documented by CSBB and Roberts et al. (1996), is also seen here when comparing EXP-H and EXP- $\mathrm{H}_{\mathrm{P}}$ [see Griffies et al. (2000a) for a review of this topic]. The weaker density gradients of EXP- $\mathrm{H}_{\mathrm{P}}$ as compared with EXP-H are also expressed in the surface fields (section 3b, Fig. 1 and section 3c, Fig. 4) and are in part due to the fact that fronts are better represented in the hybrid and isopycnic coordinate experiments, in which isopycnals can congregate to provide high resolution in regions of high density contrast. In addition, no rotation of the diffusive tensor along isopycnals is included in EXP- $\mathrm{H}_{\mathrm{P}}$, a feature which has been shown to significantly reduce numerically induced diapycnal diffusion (Griffies et al. $1998,2000 b)$. The main purpose of $E X P-H_{P}$ is to illustrate that the generalized coordinate aspect of HYCOM works well by comparing EXP- $\mathrm{H}_{\mathrm{P}}$ with similar experiments such as the GFDL-CME experiment discussed in CSBB, which also did not include any tensor rotation. In the hybrid experiments, during most months of the year, the fixed coordinates are confined to the mixed layer, where there is no need for tensor rotation. During summer, fixed coordinates extending at most 20-30 m below the mixed layer depth in the subtropical gyre can indeed be found in the hybrid model results.

The density front associated with the northeastwardflowing North Atlantic Current is well defined in the hybrid experiments by isopycnals rising sharply from about $1000 \mathrm{~m}$ to the surface (Figs. 6a,c,d). As discussed in reference to the surface circulation (section $3 b$ ), the actual location of the front differs among the hybrid experiments. In EXP-H $\sigma_{2}$ (Fig. 6c), the isopycnals are relatively flat in the $50^{\circ}-55^{\circ} \mathrm{N}$ latitude range, reflecting the due-north path of the NAC along $35^{\circ} \mathrm{W}$ in that experiment (Fig. 1). EXP-H $\sigma_{2}^{*}$ (Fig. 6d) more closely resembles EXP-H (Fig. 6a) in the region of the NAC eastward and northward turn, both in Fig. 1 and in Fig. 6. In EXP-H $\sigma_{2}$ (Fig. 6c), the surface circulation is the result of inaccurate pressure gradients at the surface [reference pressure at $20 \mathrm{MPa}(\sim 2000 \mathrm{~m})$ ], which, in the absence of any representation of thermobaric effects to account for density variations along potential isopycnals, leads to an intensified subpolar gyre and a NAC path that extends farther to the east before turning north. The mode water representation centered at $40^{\circ} \mathrm{N}$ above $500-\mathrm{m}$ depth is also strongly dependent upon the position and strength of the NAC (Figs. 6a,c,d).

We now further investigate the impact of the reference pressure choice and of thermobaricity by plotting the density difference between pairs of experiments displayed with the same reference pressure. In Fig. 6, the 

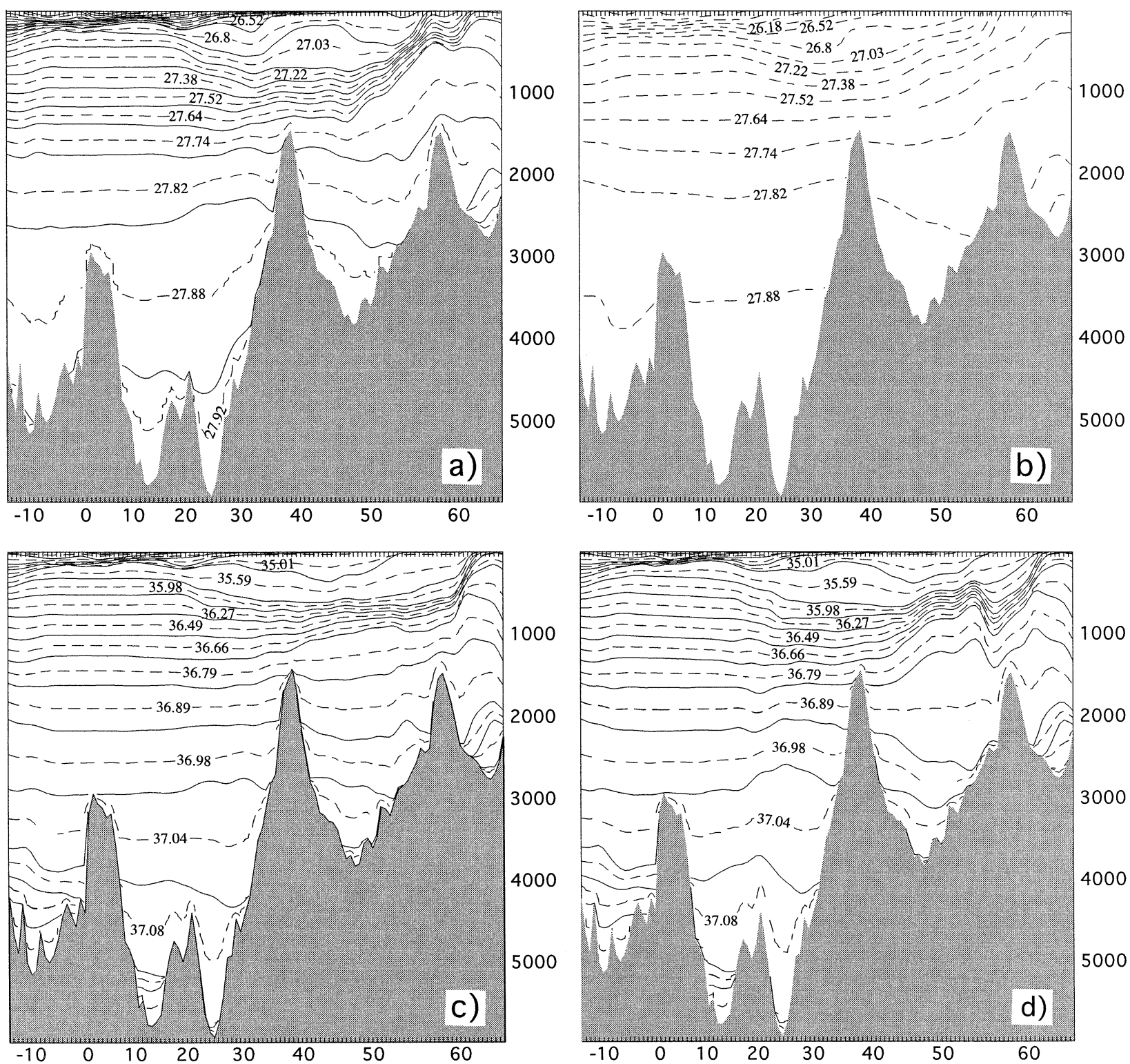

FIG. 6. Vertical cross sections along $31.8^{\circ} \mathrm{W}$, mean years 18-20: (a) EXP-H, (b) EXP- $\mathrm{H}_{\mathrm{P}}$, (c) EXP-H $\sigma_{2}$, and (d) EXP-H $\sigma_{2}^{*}$. Solid lines are layer interfaces for EXP-H, $\mathrm{H} \sigma_{2}$, and $\mathrm{H} \sigma_{2}^{*}$. Dashed lines show layer density ( $\sigma_{\theta}$ for EXP-H, $\mathrm{H}_{\mathrm{P}} ; \sigma_{2}$ for EXP-H $\left.\sigma_{2}, \mathrm{H} \sigma_{2}^{*}\right)$. Depth is shown in meters on vertical axis.

cross sections are displayed in the "native" coordinates for each experiment, that is, $\sigma_{\theta}$ for EXP-H and $\sigma_{2}$ for EXP- $\mathrm{H} \sigma_{2}, \mathrm{H} \sigma_{2}^{*}$. In Fig. 7, we show the difference between EXP-H $\sigma_{2}$ and EXP-H, first viewed in $\sigma_{\theta}$ space (Fig. 7a) and then in $\sigma_{2}$ space (Fig. 7b). Fig. 7c then shows the density difference between EXP-H $\sigma_{2}^{*}$ and EXP- $\mathrm{H} \sigma_{2}$, both displayed in their "native" $\sigma_{2}$ coordinate.

Conversion to $\sigma_{2}$ coordinates for EXP-H, or to $\sigma_{\theta}$ for EXP- $\sigma_{2}$, does not significantly alter the density distribution in the upper $1000 \mathrm{~m}$, south of $20^{\circ} \mathrm{N}$ (Figs. 7a,b). North of $55^{\circ} \mathrm{N}$, both figures indicate lighter water present in EXP-H $\sigma_{2}$, where the NAC has carried relatively warm water northward before turning east (Fig. 1). In EXP-H, however, the NAC turns north at a point farther to the west than in EXP-H $\sigma_{2}$ and then turns sharply to the east, confining cold subpolar water to higher latitudes along this section. From $\sim 25-50^{\circ} \mathrm{N}$, denser water is present between 500 and $1000 \mathrm{~m}$ in EXP- $\mathrm{H} \sigma_{2}$ relative to EXP-H due to the deeper subtropical gyre thermocline of EXP-H as seen in Fig. 6a. Water below $\sim 2000$ $\mathrm{m}$ appearing heavier in EXP- $\mathrm{H} \sigma_{2}$ relative to EXP-H when viewed in $\sigma_{2}$ (Fig. 7b) (and the opposite when viewed in $\sigma_{\theta}$; Fig. 7a) arises from the different initial conditions between the two experiments.

Figure $7 \mathrm{c}$ shows the density difference between EXP- 

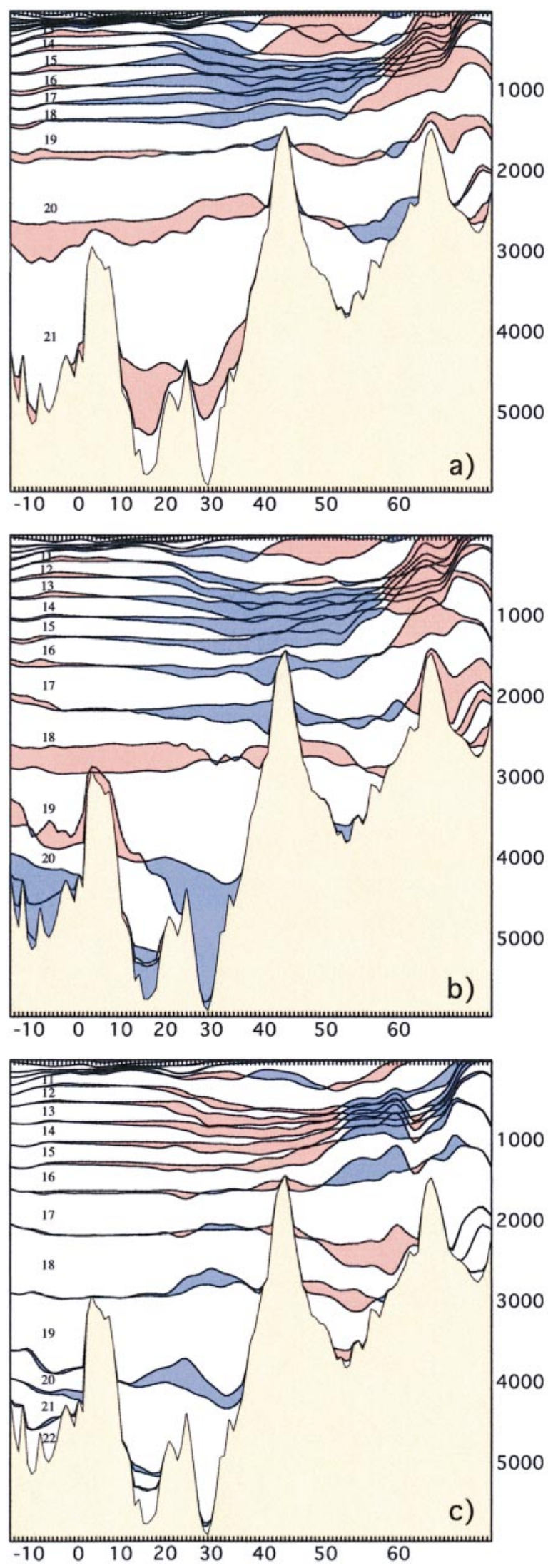

$\mathrm{H} \sigma_{2}^{*}$ and EXP- $\mathrm{H} \sigma_{2}$ displayed in $\sigma_{2}$ coordinates. The differences are due to the inclusion of thermobaric effects in EXP-H $\sigma_{2}^{*}$ and are expected in regions of steep density gradients and at depths where AABW flows northward beneath the southward-flowing North Atlantic Deep Water. Indeed, the differences between EXP$\mathrm{H} \sigma_{2}^{*}$ and $\mathrm{EXP}-\mathrm{H} \sigma_{2}$ are largest in the representation of the NAC (as noted above) and below $2000 \mathrm{~m}$. The impact of thermobaricity on the deep circulation will be further discussed in section $3 \mathrm{~g}$ by examining horizontal flow patterns below $2000 \mathrm{~m}$.

\section{2) At the equator}

Proper representation of the dynamics of the equatorial region in a numerical ocean model, in particular the strength and extent of the modeled Equatorial Undercurrent (EUC), also depends strongly on the details of the initial vertical stratification and on the vertical mixing scheme employed (Schott and Böning 1991; Blanke and Delecluse 1993). The EUC is a robust feature in all of the present experiments. Cross sections of the upper $250 \mathrm{~m}$ are presented in Fig. 8 (along $31.8^{\circ} \mathrm{W}$, crossing the equator) and in Fig. 9 (along the equator) for three of the experiments, EXP-H, EXP- $\mathrm{H}_{\mathrm{P}}$, and EXP$\mathrm{H} \sigma_{2}^{*}$, for the month of March in model year 20. The left panels show contours of density overlaid on the vertical coordinates ( $\sigma_{\theta}$, pressure, $\sigma_{2}$, respectively); the right panels are overlaid with contours of zonal velocity. The core EUC velocity is $\sim 40 \mathrm{~cm} \mathrm{~s}^{-1}$ in all three experiments, roughly half the core speed given by Garzoli and Katz (1983) as a 5 -month average at $23.5^{\circ} \mathrm{W}$. The EUC strength is strongly sensitive to horizontal resolution and to the choice of mixing parameterization.

Increased vertical discretization in the upper $300 \mathrm{~m}$, by a factor of 2, is evident in the hybrid experiments EXP-H and EXP-H $\sigma_{2}^{*}$ in both zonal and meridional sections of density (Figs. 8a,e and 9a,e), when compared to the fixed-coordinate EXP-H $\mathrm{H}_{\mathrm{P}}$ (Figs. 8c and 9c). The greater resolution is particularly evident in EXP-H since the vertical discretization in EXP-H $\sigma_{2}^{*}$ has been mapped to provide more resolution near the bottom. In further comparing the fixed-coordinate and hybrid experiments, we note that 1) the southern branch of the South Equatorial Current (SEC) is surface intensified in EXP-H and EXP-H $\sigma_{2}^{*}$ (Figs. 8b,f) as compared with that of EXP$\mathrm{H}_{\mathrm{P}}$ (Fig. 8d), most likely as a result of the lack of nearsurface resolution in $\mathrm{EXP}-\mathrm{H}_{\mathrm{P}}$; and 2) the representations of the westward cross-equatorial flows associated with the North Brazil Current differ at the undercurrent level:

$\leftarrow$

FIG. 7. Vertical cross sections along $31.8^{\circ} \mathrm{W}$, mean years $18-20$, showing density difference between two experiments: (a) EXP-H $\sigma_{2}-$ EXP-H, displayed in $\sigma_{\theta}$; (b) EXP-H $\sigma_{2}-$ EXP-H, displayed in $\sigma_{2}$; and (c) EXP-H $\sigma_{2}^{*}-$ EXP-H $\sigma_{2}$, displayed in $\sigma_{2}$. Red (blue) shading indicates first experiment is lighter (heavier) than second experiment. 

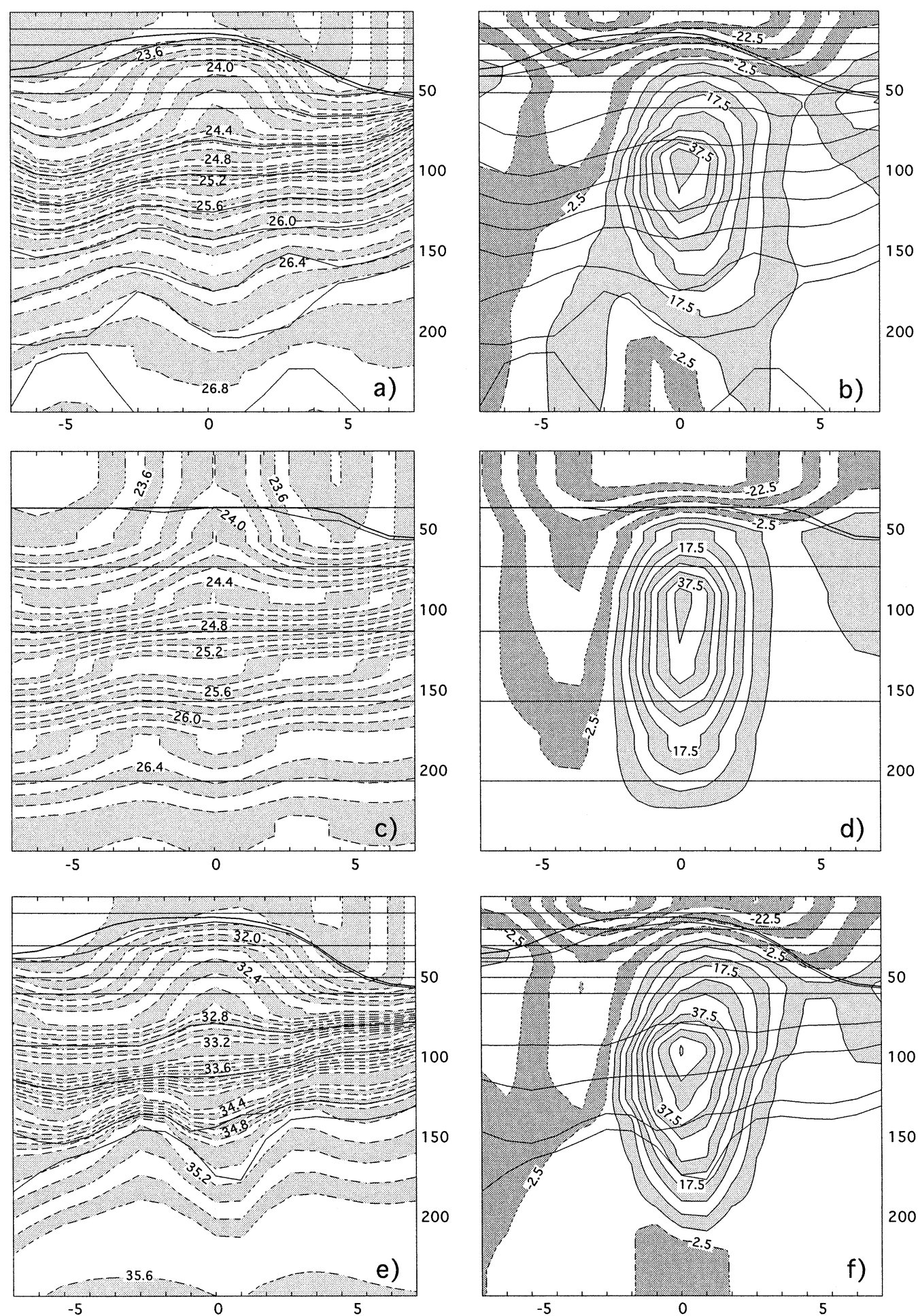

Fig. 8. Vertical cross sections along $31.8^{\circ} \mathrm{W}$ for $\sim 7^{\circ} \mathrm{S}-7^{\circ} \mathrm{N}$, Mar year 20 , showing contours of density (left: dashed contours, contour interval 0.1) and zonal velocity [right: solid (dashed) contours are positive (negative) velocity, contour interval $5 \mathrm{~cm} \mathrm{~s}^{-1}$ ]. (a),(b) EXP-H $\left(\sigma_{\theta}\right)$; (c),(d) EXP- $\mathrm{H}_{\mathrm{P}}\left(\sigma_{\theta}\right)$; (e),(f) EXP-H $\sigma_{2}^{*}\left(\sigma_{2}\right)$. Solid lines are layer interfaces. Depth shown in meters on vertical axis. 

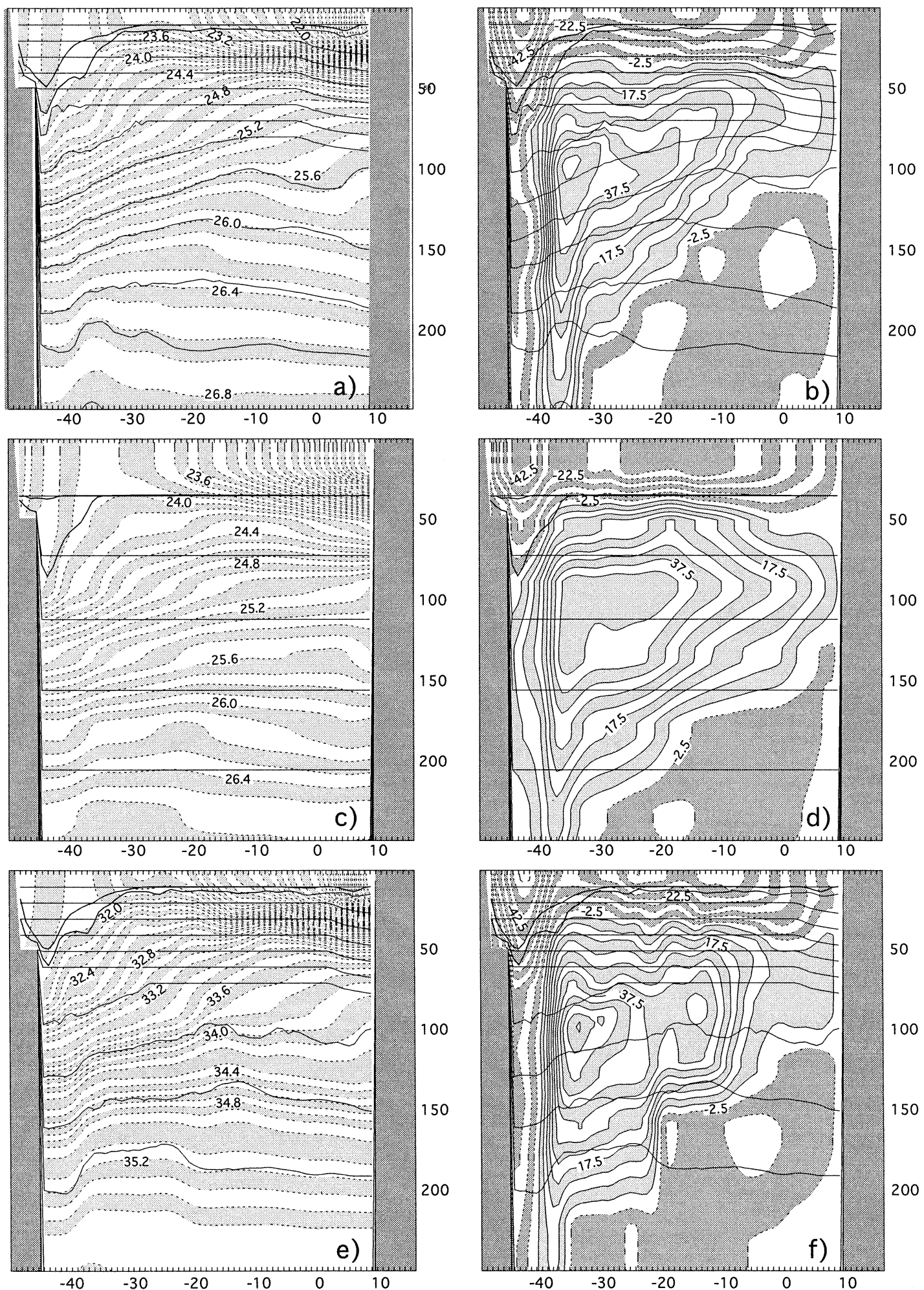

FIG. 9. Vertical cross sections along the equator across the model basin for Mar year 20 showing contours of density (left: dashed contours, contour interval 0.1 ) and zonal velocity [right: solid (dashed) contours are positive (negative) velocity, contour interval $5 \mathrm{~cm} \mathrm{~s}^{-1}$ ]. (a),(b) EXP-H $\left(\sigma_{\theta}\right)$; (c),(d) EXP-H $\mathrm{H}_{\mathrm{P}}\left(\sigma_{\theta}\right)$; (e),(f)EXP-H $\sigma_{2}^{*}\left(\sigma_{2}\right)$. Solid lines are layer interfaces. Depth is shown in meters on vertical axis. 
there is no westward flow below $100 \mathrm{~m}$ in $\mathrm{EXP}_{\mathrm{P}} \mathrm{H}_{\mathrm{P}}(\mathrm{Fig}$. $9 \mathrm{~d})$ in contrast with the cross-equatorial flow in EXP$\mathrm{H}$ and EXP-H $\sigma_{2}^{*}$ (Figs. 9b,f), which extends to a depth of $250 \mathrm{~m}$. There is, however, very little difference in the flow representation as a consequence of reference pressure choice with the correction for thermobaricity $\left(\mathrm{EXP}-\mathrm{H} \sigma_{2}^{*}\right)$ or without that correction $\left(\mathrm{EXP}-\mathrm{H} \sigma_{2}\right.$, not illustrated). This is the expected result since the impact of thermobaricity is felt more strongly in regions of steep isopycnals (Sun et al. 1999).

\section{e. Time evolution of water masses}

Analysis of the cross sections along $31.8^{\circ} \mathrm{W}$ in the previous section allowed us to document some of the differences in model results that arise from the choice of model configuration. That analysis does not, however, provide any indication of the time evolution of the different water masses initially present in the simulations. For that purpose, we monitor in time the amount of mass in 16 density classes that approximately correspond to the 16 heaviest coordinate densities in the $\sigma_{\theta}$ and $\sigma_{2}$ experiments (see Table 2 for details).

The time evolution (years 1-20) of the 16 domainaveraged layer thicknesses corresponding to the 16 density classes is shown in Fig. 10 for experiments $\mathrm{M}, \mathrm{H}_{\mathrm{KT}}$, $\mathrm{H}, \mathrm{H}_{\mathrm{P}}, \mathrm{H} \sigma_{2}$, and $\mathrm{H} \sigma_{2}^{*}$. When multiplied by the surface domain area, the averaged layer thickness represents the volume of water corresponding to each density class. Only the September averaged layer thicknesses are plotted in order to remove the seasonal signal. The bottom curve in each panel of Fig. 10 shows the time evolution of the heat content, defined as

$$
\rho c_{p} \sum T h \Delta x^{2} / \sum \Delta x^{2} \quad \text { (units } \mathrm{J} \mathrm{m}^{-2} \text { ), }
$$

where $\rho=1000 \mathrm{~kg} \mathrm{~m}^{-3}, c_{p}=3990 \mathrm{~J} \mathrm{~kg}^{-1} \mathrm{~K}^{-1}, T$ is temperature, $h$ is layer thickness, $\Delta x$ is horizontal grid size, and the sums are taken over all horizontal grid points in all layers.

The layer thickness trends shown in Figs. 10a-c for the $\sigma_{\theta}$ experiments [EXP-M (MICOM), EXP- $\mathrm{H}_{\mathrm{KT}}(\mathrm{HY}-$ COM with $\mathrm{K}-\mathrm{T}$ mixed layer), and EXP-H (HYCOM with KPP vertical mixing)] are highly similar, indicating that the interior mass distribution is not unduly dependent upon either the mixed layer architecture (which can lead to large temporal changes in basin-averaged layer thickness) or the vertical coordinate choice (hybrid versus isopycnic). In the deepest layer of these experiments, the volume remains nearly constant over time. In contrast, EXP-H $\mathrm{H}_{\mathrm{P}}$ (Fig. 10d), configured with fixed coordinate levels, shows the loss of nearly all of the densest (and coldest) water in the domain over the 20yr period, a loss that is reflected in the greater increase in heat content seen for this experiment. These results again illustrate the divergence over time in deep-water mass structures between models formulated in depth and isopycnic coordinates (CSBB; Roberts et al. 1996; Griffies et al. 2000a; Willebrand et al. 2001).
The changes over time in water mass distribution in the two $\sigma_{2}$ experiments are much less than in the $\sigma_{\theta}$ experiments. The five deepest layers remain relatively constant in basin-averaged thickness over 20 years in both EXP-H $\sigma_{2}$ and EXP-H $\sigma_{2}^{*}$ (Figs. 10e,f). While the mid- and upper-layer thickness evolution differs slightly between the two experiments, the time-mean basinwideaveraged layer thickness remains similar for corresponding layers. In contrast with the increasing heat content seen in the $\sigma_{\theta}$ experiments, the basin-averaged heat content decreases slightly over 20 years in EXP-H $\sigma_{2}$ and remains relatively steady in EXP-H $\sigma_{2}^{*}$, the experiment with thermobaricity.

\section{f. Overturning and heat transport}

The meridional overturning cell and associated heat transport, which are driven by the water mass transformations that take place both inside and outside the buffer zones, serve as good indicators of the model's performance. Comparison of the meridional overturning streamfunctions for experiments EXP-H (with hybrid pressure-isopycnal coordinate surfaces) and $\mathrm{EXP}-\mathrm{H}_{\mathrm{P}}$ (with fixed levels) (Figs. 11a,b) shows the strong impact of the coordinate choice. EXP- $\mathrm{H}_{\mathrm{P}}$ exhibits a maximum overturning of $13 \mathrm{~Sv}$, centered north of $45^{\circ} \mathrm{N}$, in contrast with a maximum $17 \mathrm{~Sv}$ for EXP-H reaching the southern boundary. Below $3000 \mathrm{~m}$, the southward flow of EXP$\mathrm{H}_{\mathrm{P}}$ is much weaker (maximum $5 \mathrm{~Sv}$ ) than the $9 \mathrm{~Sv}$ reaching the southern boundary at depth in EXP-H. A similar result was obtained by CSBB when comparing the GFDL and MICOM experiments. As noted in section $3 \mathrm{~d}(1)$, there is no rotation of the diffusive tensor along isopycnals in EXP- $\mathrm{H}_{\mathrm{P}}$. Rotation of the tensor, together with the inclusion of a parameterization of the effects of mesoscale eddies on the transport of tracers (Gent and McWilliams 1990; Gent et al. 1995; McDougall and McIntosh 2001), increases the strength and extent of the overturning circulation in depth-coordinate models (CSBB; Böning et al. 1995).

The overturning streamfunctions for experiments in $\sigma_{2}$ coordinates, EXP-H $\sigma_{2}$ and EXP-H $\sigma_{2}^{*}$ (Figs. 11c,d), are quantitatively similar to that of EXP-H (Fig. 11a) in terms of strength of the circulation, with maxima between 17 and 19 Sv. The near-surface overturning cell is slightly stronger in EXP-H $\sigma_{2}$ than in EXP-H and $\mathrm{EXP}-\mathrm{H} \sigma_{2}^{*}$, reflecting the stronger surface circulation in EXP-H $\sigma_{2}$ that results from inaccurate pressure gradients at the surface (Fig. 1). The pattern of the overturning, however, differs significantly in EXP-H $\sigma_{2}$ and EXP$\mathrm{H} \sigma_{2}^{*}$ from that of EXP-H in two respects: first, the maximum in transport is centered around the equator in EXP-H as compared with $\sim 40^{\circ} \mathrm{N}$ in EXP-H $\sigma_{2}$ and EXP$\mathrm{H} \sigma_{2}^{*}$ and, second, the dense water flows south at shallower depths in EXP-H $\sigma_{2}$ and EXP-H $\sigma_{2}^{*}$ than in EXP$\mathrm{H}$. The circulation is weaker at $2000 \mathrm{~m}$ in the two $\sigma_{2}$ experiments, and the signature of the AABW (not present in the $\sigma_{\theta}$ vertical discretization) begins to appear at 
a)

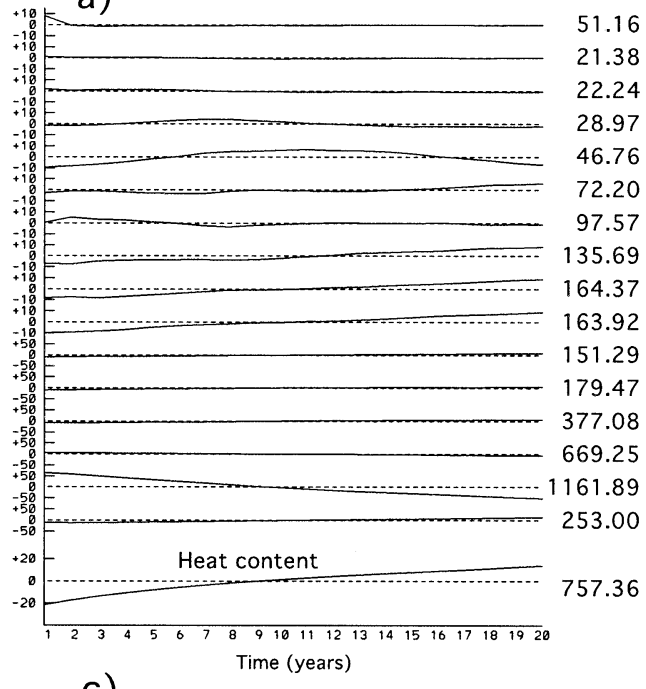

c)

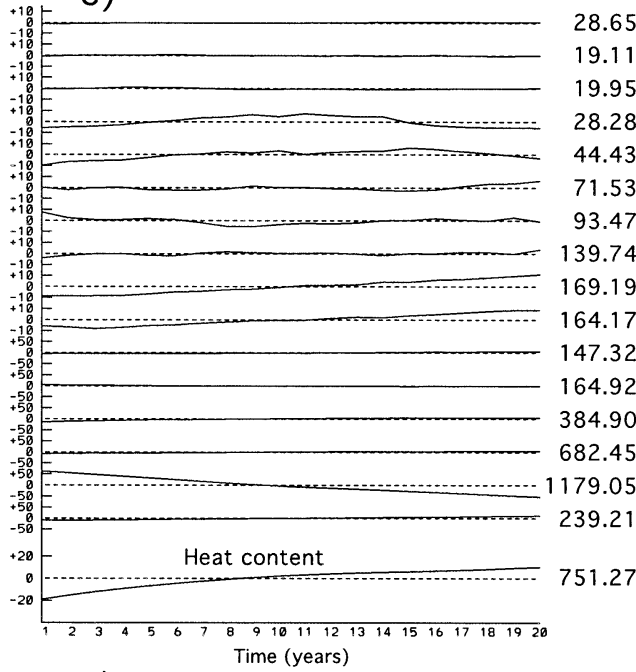

e)

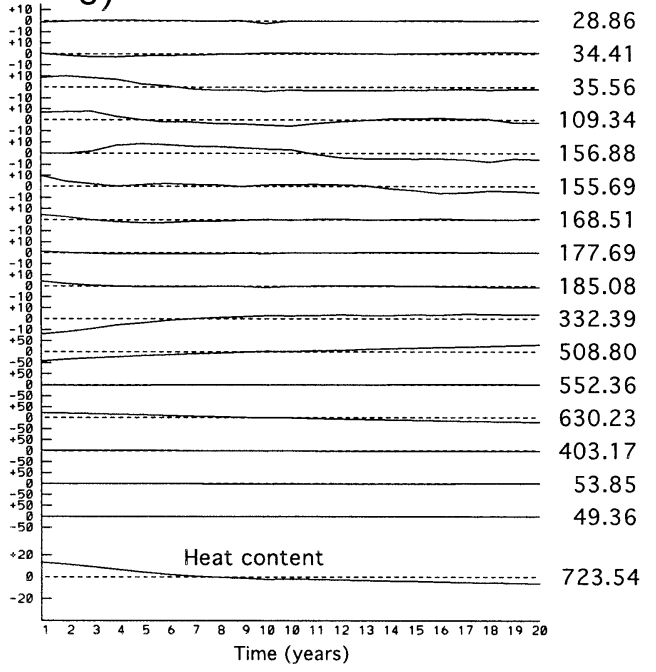

b)

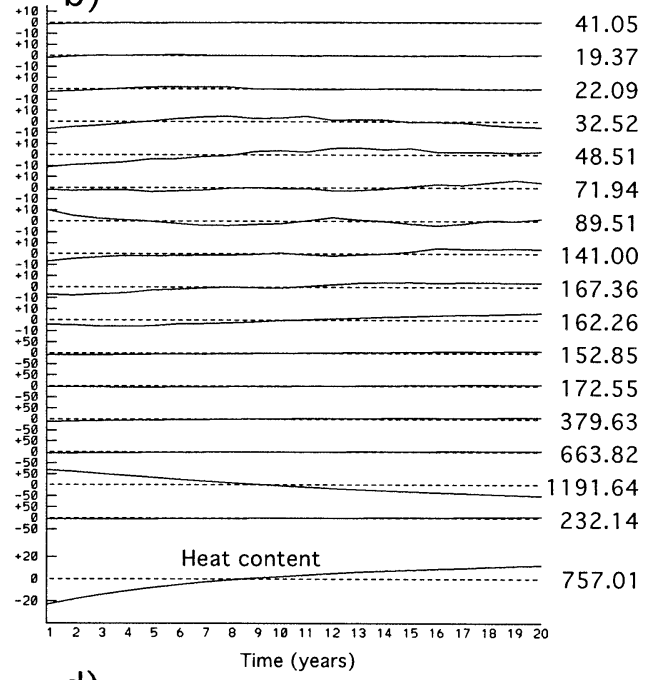

d)

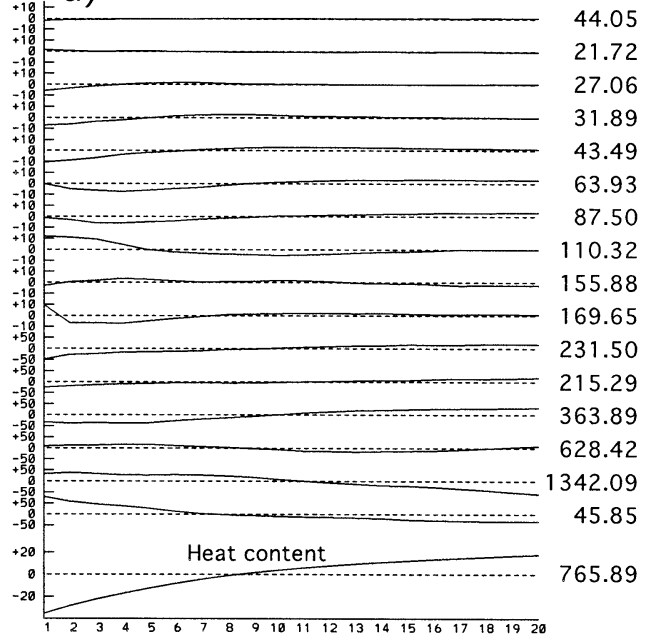

f) Time (years)

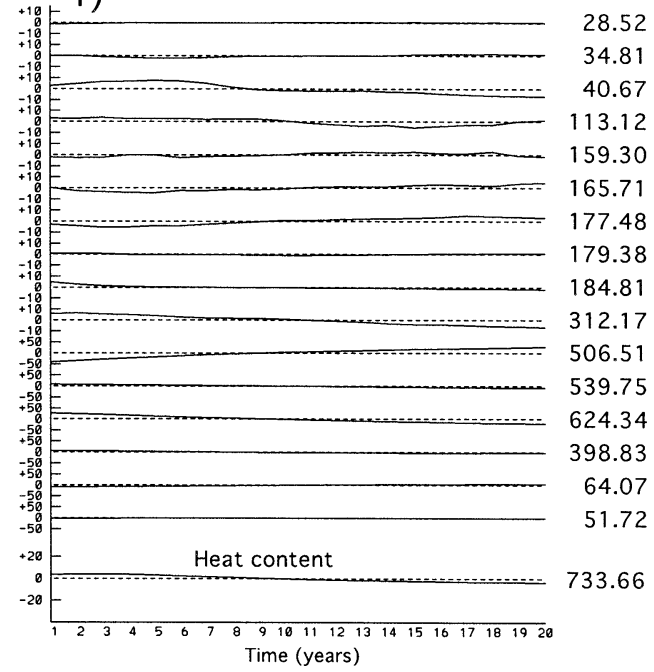

FIG. 10. 20-yr time evolution of the domain-averaged layer thicknesses in meters for 16 density classes chosen as described in the text and of the domain-averaged heat content (bottom curve), computed for the month of September for (a) EXP-M, (b) EXP- $\mathrm{H}_{\mathrm{KT}}$, (c) EXP-H, (d) EXP- $\mathrm{H}_{\mathrm{P}}$, (e) EXP-H $\sigma_{2}$, and (f) EXP-H $\sigma_{2}^{*}$. Basin-mean time-mean thickness and heat content values are shown at right. Tick marks on left axis indicate difference relative to time-mean value. 

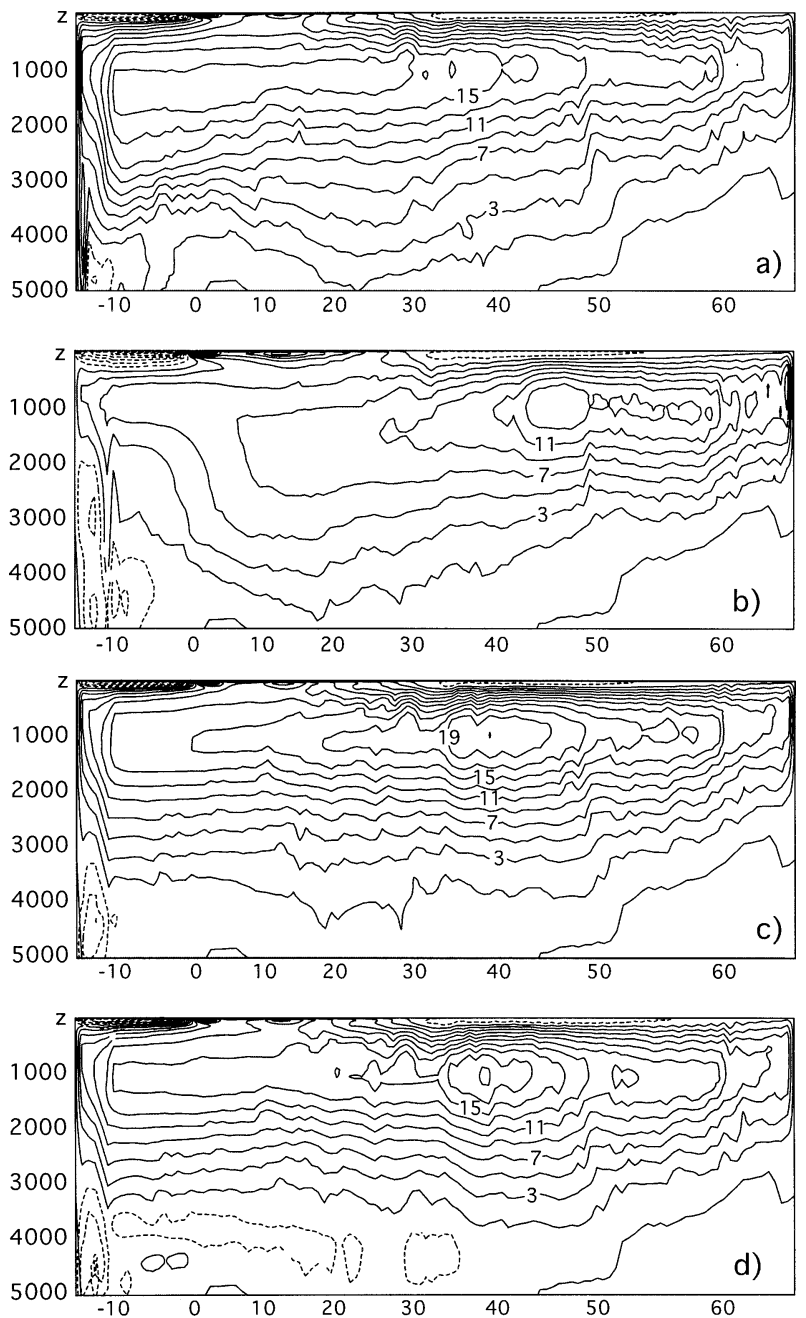

FIG. 11. Meridional overturning streamfunction, mean years 18

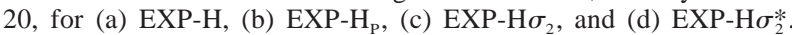
Contour interval is $2 \mathrm{~Sv}$; the zero contour is not shown. Dashed contours indicate negative values. Depth is shown in meters on vertical axis. Latitude is shown on horizontal axis.

the deepest levels. It remains at the southern boundary in EXP-H $\sigma_{2}$, while in EXP-H $\sigma_{2}^{*}$, where the pressure gradient term in the horizontal momentum equation is modified to account for the effect of thermobaricity, the $\mathrm{AABW}$ is seen to flow north from the southern boundary to $\sim 25^{\circ}-35^{\circ} \mathrm{N}$.

These differences in overturning circulation can be seen more clearly by examining the zonally averaged meridional transport below $2000 \mathrm{~m}$ for EXP-H, $\mathrm{H}_{\mathrm{P}}$, $\mathrm{H} \sigma_{2}$, and $\mathrm{H} \sigma_{2}^{*}$ (Fig. 12). This deep transport is similar for experiments $\mathrm{H}, \mathrm{H} \sigma_{2}$, and $\mathrm{H} \sigma_{2}^{*}$ north of $\sim 35^{\circ} \mathrm{N}$. South of that latitude, EXP-H $\sigma_{2}$ and EXP-H $\sigma_{2}^{*}$ show lower transport below $2000 \mathrm{~m}$ than is seen in EXP-H. In these two cases (Figs. 11c,d), the strongest southward flow, south of $\sim 35^{\circ} \mathrm{N}$, lies at shallower depths than in EXP-H due to the northward intrusion of AABW just above the bottom. EXP-H exhibits the maximum

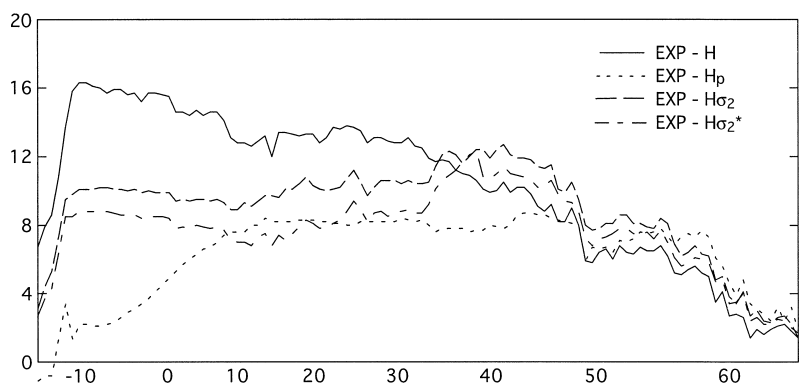

FIG. 12. Zonally averaged meridional overturning transport below $2000 \mathrm{~m}$, mean years 18-20. Vertical axis is in Sverdrups. Latitude is shown on horizontal axis.

amount of deep southward flow among these experiments, and $\mathrm{EXP}-\mathrm{H}_{\mathrm{P}}$ (with strong upwelling) the least, consistent with the flow patterns shown in Figs. 11a and $11 \mathrm{~b}$.

The mean model heat transport for years 18-20 (Fig. 13a) clearly shows the relatively low transport values

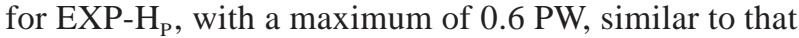
found in the GFDL CME experiment without parameterization of the eddy-induced "bolus" transport (Gent and McWilliams 1990; Gent et al. 1995; Böning et al., 1995; CSBB; McDougall and McIntosh 2001). For the remaining experiments, the order from lowest (1.0 PW) to highest $(1.3 \mathrm{PW})$ maximum heat transport is EXP$\mathrm{M}, \mathrm{H}_{\mathrm{KT}}, \mathrm{H} \sigma_{2}^{*}, \mathrm{H}, \mathrm{H} \sigma_{2}$. That is, the hybrid experiments with KPP vertical mixing have higher heat transport than those with K-T mixed layer. However, the transport values for all experiments are in reasonable agreement with the $1.25 \mathrm{PW}$ estimated at $25^{\circ} \mathrm{N}$ by Hall and Bryden (1982) and with the $1.1 \mathrm{PW}$ indicated at $30^{\circ} \mathrm{N}$ by the inverse calculation of Macdonald and Wunsch (1996).

The January and July variations in heat transport as a function of latitude, for the average of model years 18-20 (Fig. 13b), show the same order of lowest to highest transport values among the experiments as was seen for the annual mean transport. In both months, the hybrid KPP vertical mixing cases EXP-H, $\mathrm{H} \sigma_{2}, \mathrm{H} \sigma_{2}^{*}$ produce higher heat transport than those with $\mathrm{K}-\mathrm{T}$ mixed layer (EXP-M, $\mathrm{H}_{\mathrm{KT}}$ ), with the lowest maximum transport values being exhibited by EXP- $\mathrm{H}_{\mathrm{P}}$. However, the annual variation of heat transport across $8^{\circ} \mathrm{N}$, which is sensitive to both the model wind stress and grid resolution (Böning and Herrmann 1994), is seen to be $\sim 1.5$ $\mathrm{PW}$ in all experiments, including EXP- $\mathrm{H}_{\mathrm{P}}$. This result was also found by CSBB (their Fig. 8) for GFDL and MICOM experiments having similar grid resolution and forced by the same wind stress data.

The order of lowest to highest heat transport values among the KPP experiments $\left(\mathrm{EXP}-\mathrm{H}_{\mathrm{P}}, \mathrm{H} \sigma_{2}^{*}, \mathrm{H}, \mathrm{H} \sigma_{2}\right)$ is also replicated in figures of the 3-yr cycle of heat transport for those experiments at $25^{\circ} \mathrm{N}$ and at $40^{\circ} \mathrm{N}$ (Figs. 14a,b). The highest maximum value at both latitudes is that of EXP- $\mathrm{H} \sigma_{2}$, which displays a summer transport maximum at $40^{\circ} \mathrm{N}$ approximately $0.2 \mathrm{PW}$ greater than the values for EXP-H and EXP-H $\sigma_{2}^{*}$. At 

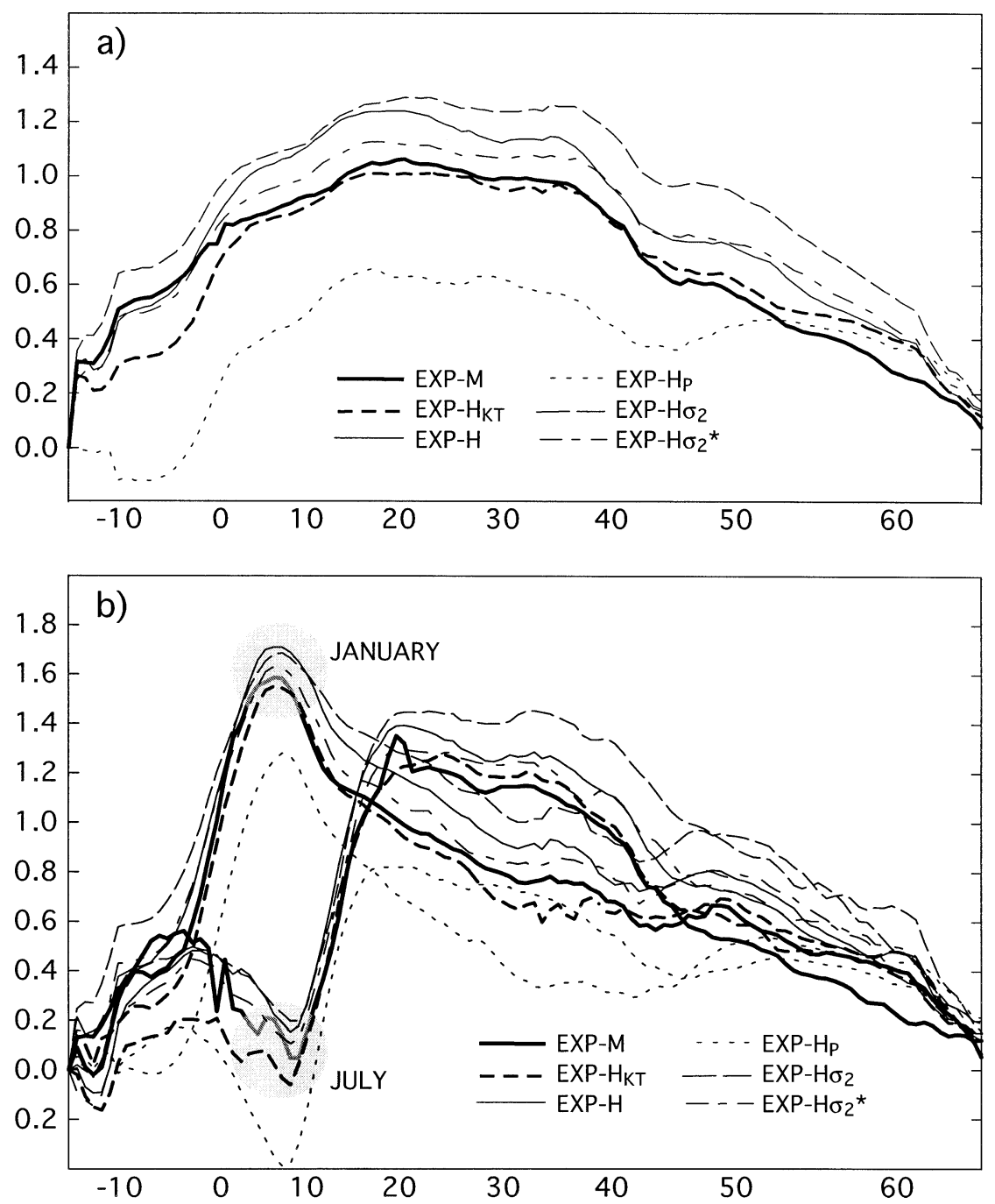

FIG. 13. (a) Meridional heat transport, mean years 18-20; (b) meridional heat transport, Jan mean and Jul mean, years 18-20; extrema highlighted. Vertical axis is in petawatts. Latitude is shown on horizontal axis.

$25^{\circ} \mathrm{N}$, the difference between the maximum summer value for EXP-H $\sigma_{2}$ and those of the other hybrid KPP experiments is not as large. The highest heat transport values at $40^{\circ} \mathrm{N}$ in EXP- $\mathrm{H} \sigma_{2}$ are due to a greater contribution to the transport from the wind-driven gyre component at that latitude, resulting from the stronger surface circulation in EXP-H $\sigma_{2}$ (section $3 b$ ).

\section{g. Deep-water mass transport}

A significant difference between results of the $\sigma_{\theta}$ and $\sigma_{2}$ coordinate experiments is the model representation of AABW. This is not surprising since there is no distinct coordinate layer representing $\mathrm{AABW}$ in a $\sigma_{\theta}$ discretization: one cannot have a monotonically increasing $\sigma_{\theta}$ distribution in the vertical in the presence of AABW due to the folding of $\sigma_{\theta}$ isopycnals in the density range of that water mass. However, this is not the case when the reference pressure is set to $20 \mathrm{MPa}(\sim 2000 \mathrm{~m})$.

The meridional overturning streamfunctions for the three experiments-EXP-H ( $\sigma_{\theta}$ coordinate), EXP-H $\sigma_{2}$ $\left(\sigma_{2}\right.$ coordinate $)$, and EXP-H $\sigma_{2}^{*}\left(\sigma_{2}\right.$ coordinate with thermobaricity) - do indeed show that adoption of a $\sigma_{2}$ coordinate renders the model results more realistic by permitting representation of the AABW circulation in the deep ocean (Figs. 11a,c,d). Furthermore, the incorporation of thermobaricity in $\mathrm{EXP}-\mathrm{H} \sigma_{2}^{*}$ increases the strength of the overturning cell associated with the AABW (Fig. 11d).

In this section, we further investigate the impact of the reference pressure choice and thermobaricity on the deep ocean by examining the horizontal flow patterns of the "lower deep" density class [as defined by Schmitz (1996): $\sigma_{\theta}>27.8$; roughly corresponding to $\left.\sigma_{2}>36.7\right]$ 

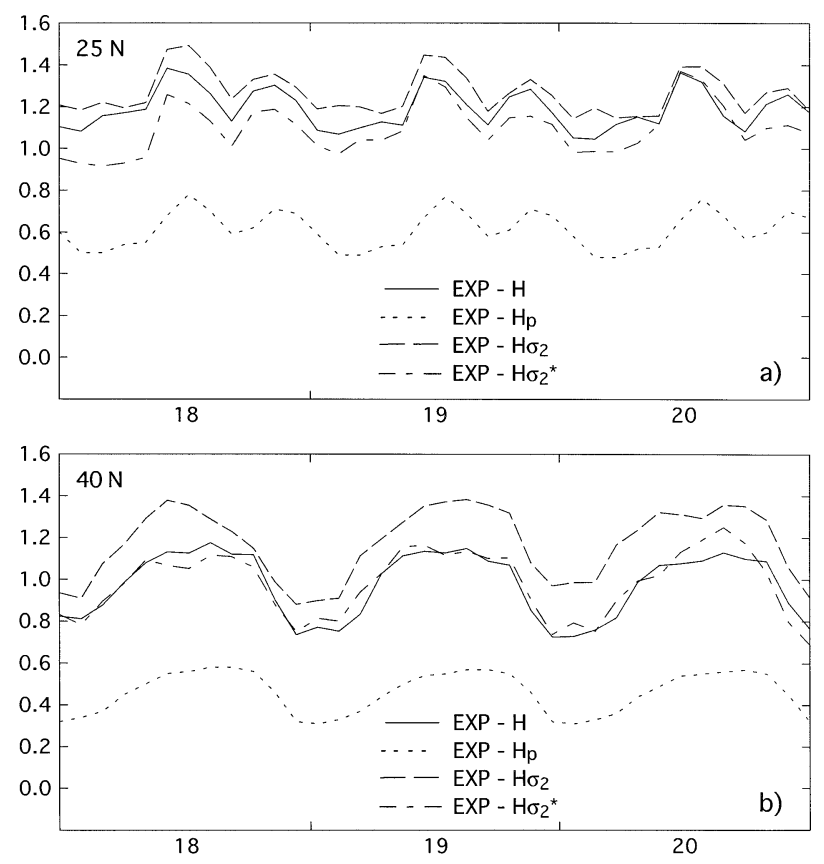

FIG. 14. Annual cycle of meridional heat transport for model years $18-20$ at $25^{\circ}$ and $40^{\circ} \mathrm{N}$. Vertical axis is in petawatts.

for experiments $\mathrm{H}, \mathrm{H} \sigma_{2}$, and $\mathrm{H} \sigma_{2}^{*}$. For each experiment, the transport functions for the water masses belonging to the "lower deep" class (Fig. 15) are calculated by summing up northward fluxes across each latitude line.

With a reference pressure at $20 \mathrm{MPa}(\sim 2000 \mathrm{~m})$ and no correction for thermobaric effects, EXP-H $\sigma_{2}$ exhibits a stronger circulation in the deep ocean than EXP-H, just as previously seen at the surface (Fig. 1; section $3 \mathrm{~b})$. The total southward deep transport is a maximum in EXP-H $\sigma_{2}$, relative to EXP-H and EXP-H $\sigma_{2}^{*}$, with values between 17 and $19 \mathrm{~Sv}$ (Fig. 15b). EXP-H $\sigma_{2}$ also displays a more intense deep western boundary current (DWBC) than in either EXP-H or EXP-H $\sigma_{2}^{*}$. The high heat transport values in EXP-H $\sigma_{2}$ (Figs. 13, 14) result from the stronger circulation, both surface and deep. In EXP-H $\sigma_{2}^{*}$, the pressure gradients at the surface and in the deep ocean are modified to take thermobaricity into account. The end result is (i) a weaker southward deep transport (15-17 Sv; Fig. 15c) in EXP-H $\sigma_{2}^{*}$ than in EXP-H $\sigma_{2}$, and (ii) an interior circulation south of $20^{\circ} \mathrm{N}$ representative of AABW northward transport (which is nonexistent in both EXP-H and EXP-H $\sigma_{2}$, Figs. 15a,b). The three transport function figures (Fig. 15) clearly indicate each experiment's capability in regard to representation of the $\mathrm{AABW}$, as can be seen by a closer examination of the southeast corner of the basin. Only in EXP-H $\sigma_{2}^{*}$, in which the pressure gradient term in the horizontal momentum equation is modified to account for the effect of thermobaricity, can the AABW be seen flowing northward along the western side of the South Atlantic and then crossing to the eastern side of the domain north of the equator, to $\sim 20^{\circ} \mathrm{N}$.

\section{Summary and discussion}

This study has two primary goals: (i) to show the viability of a generalized (hybrid) coordinate ocean model by validating it against observations and previous simulations configured for the North and equatorial Atlantic and (ii) to document the importance of thermobaricity and the choice of reference pressure.

The first goal was addressed by analyzing a series of experiments performed with HYCOM under a variety of conditions. The generalized aspect of the model was documented by performing a few single-coordinate experiments (isopycnic, pressure level) and the hybrid aspect by performing experiments with two mixed layer parameterizations (Kraus-Turner and KPP). These experiments do indeed illustrate that the concept of a generalized (hybrid) coordinate is viable.

While the single-coordinate experiments illustrate the flexibility of the model, they bring forward some of the limitations of choosing only one coordinate. Using KPP, it is possible to run HYCOM using only isopycnic coordinates (i.e., no bulk mixed layer as in MICOM). However, the model representation of the subpolar gyre strength and of the NAC is very sensitive to the number of nonmassless (and thus active) layers in that unstratified region. The hybrid configuration is more efficient in the sense that a proper vertical discretization of the subpolar gyre can be achieved with 10-20 layers less than the number that would be required for the pure isopycnic configuration. The hybrid configuration also provides high vertical resolution in the mixed layer throughout the basin. When only pressure-level coordinates are used, the simulation exhibits the limitations (pointed out by previous model comparison exercises) of fixed versus isopycnic coordinates, that is, loss of the dense water masses and lack of vertical resolution in regions of high density contrast when fixed coordinates are used [see Griffies et al. (2000a) and Willebrand et al. (2001) for a review].

The second goal addresses a specific issue which is present in models such as HYCOM that employ potential density either as the sole vertical coordinate or as one component of the hybrid framework: the question of how best to represent buoyancy contrasts given a potential density distribution based on a uniform reference pressure. The thermobaric effect, that is, the modulation of seawater compressibility by potential temperature anomalies, must be considered in order to account for the difference between buoyancy and potential density. The questions of representation of the potential density distribution for a given reference pressure and of the importance of accounting for the thermobaric effect have been addressed in this work principally by reference to the results of three experimentsone with reference pressure at the surface $\left(\sigma_{\theta}\right.$ coordinate), one with reference pressure at $20 \mathrm{MPa}(\sim 2000$ $\mathrm{m} ; \sigma_{2}$ coordinate), and one with reference pressure at $20 \mathrm{MPa}$ and with thermobaric effects included $\left(\sigma_{2}^{*}\right)$. 

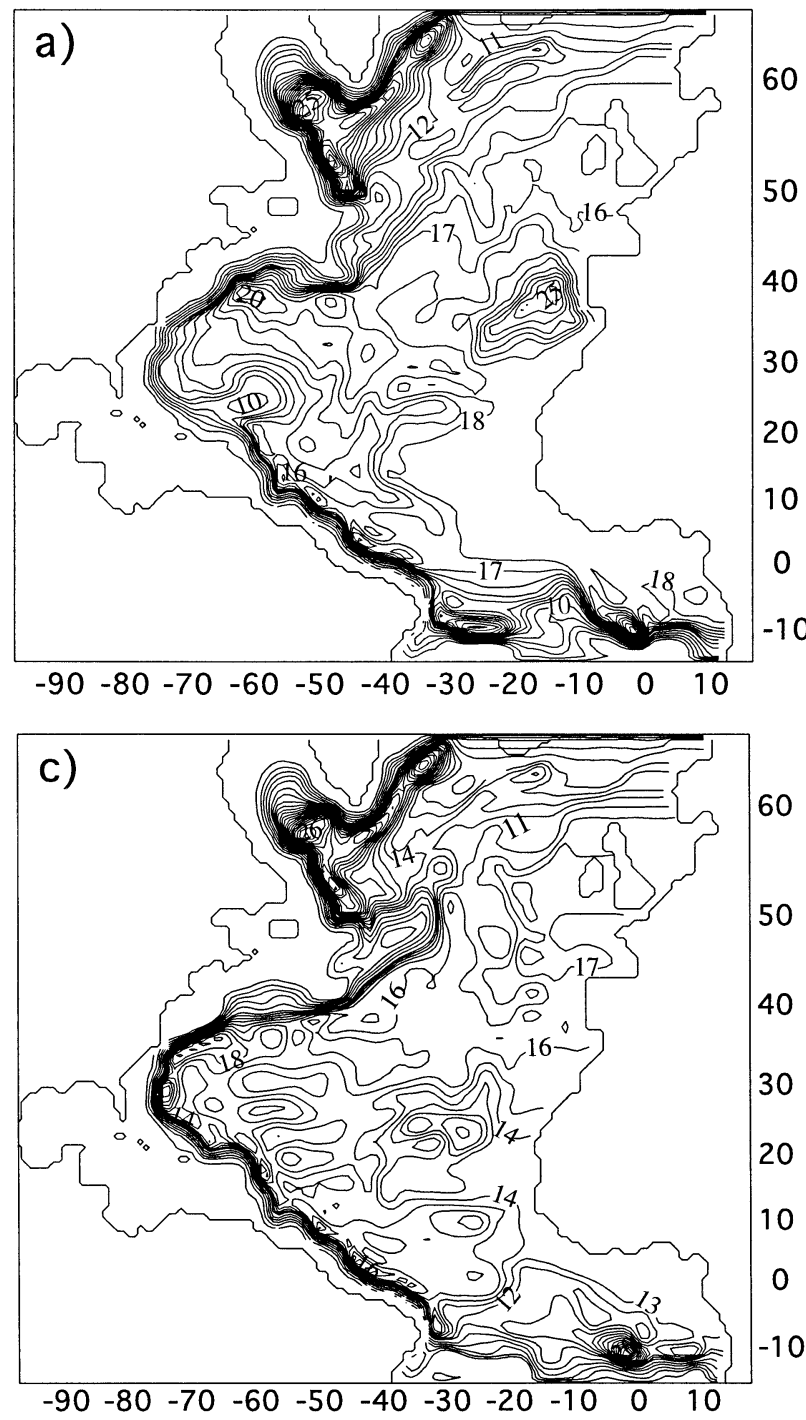

The main difference between the $\sigma_{\theta}$ and $\sigma_{2}$ experiments is the model's representation of AABW: there is no distinct water mass representing $\mathrm{AABW}$ in the $\sigma_{\theta}$ discretization since one cannot have a monotonically increasing $\sigma_{\theta}$ distribution in the vertical in the presence of AABW because of the folding of $\sigma_{\theta}$ isopycnals in the density range of that water mass. The density structure of the AABW is well represented when a reference pressure of $20 \mathrm{MPa}(\sim 2000 \mathrm{~m})$ is selected. It is important to note that there is no single reference pressure value that would not create coordinate folding somewhere in the World Ocean. However, it has been found that a reference pressure of $20 \mathrm{MPa}$ is near optimal in that regard. Furthermore, in the few places where folding might occur, the generalized-coordinate aspect of HYCOM is such that it allows interfaces to not necessarily coincide with potential isopycnals.

The importance of thermobaricity is illustrated by the differences between the $\sigma_{2}$ and $\sigma_{2}^{*}$ experiments. Without

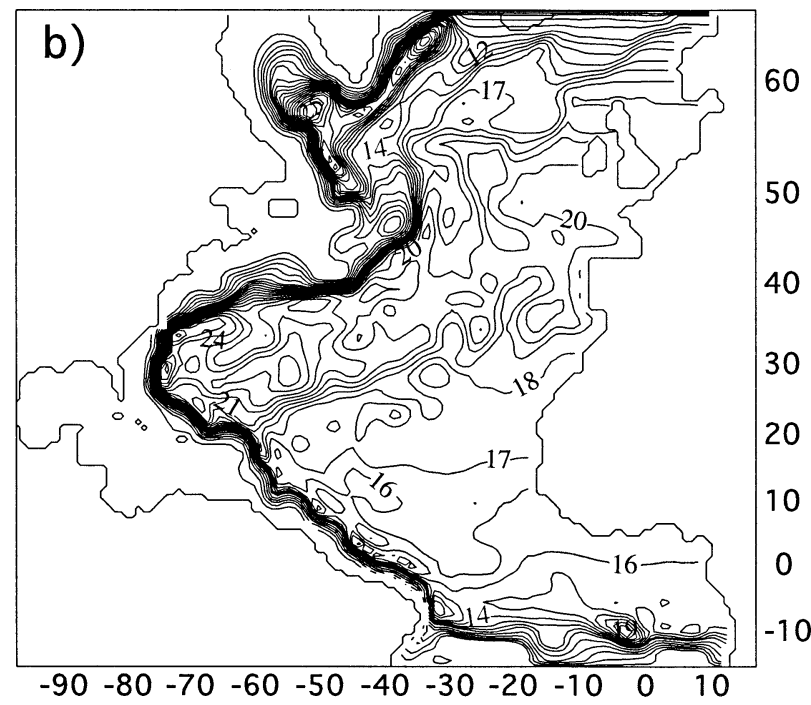

FIG. 15. Transport function for "lower deep" water masses $\left(\sigma_{\theta}>\right.$ 27.8), mean years 18-20: (a) EXP-H, (b) EXP-H $\sigma_{2}$, and (c) EXP$\mathrm{H} \sigma_{2}^{*}$. Contour interval is $1 \mathrm{~Sv}$. The zero contour is not shown.

inclusion of the thermobaric effects, the pressure gradient above and below $2000 \mathrm{~m}$ does not take into account the modulation of seawater compressibility by potential temperature anomalies. Such variations give rise to pressure forces that cannot be ignored in a general circulation model: both the surface and deep circulation are much stronger in the experiment without thermobaricity. It is also only in the $\sigma_{2}^{*}$ experiment that the AABW can be seen flowing northward on the western side of the South Atlantic and then crossing to the eastern side of the domain north of the equator, to $\sim 20^{\circ} \mathrm{N}$.

The experiments discussed in this paper not only demonstrate the versatility of the hybrid approach, but also the importance of the reference pressure choice and of the need to include thermobaricity. Further investigation of the model's performance is under way in a fine mesh $\left(1 / 12^{\circ}\right)$ North and equatorial Atlantic configuration. HY$\mathrm{COM}$ is at the center of an effort entitled "The Hybrid Coordinate Ocean Model (HYCOM) Consortium for 
Data-Assimilative Ocean Modeling" funded by the National Ocean Partnership Program (NOPP). The primary purpose of the consortium (see http://hycom.rsmas. miami.edu) is the establishment of a global real-time ocean forecast system based on HYCOM with sophisticated data assimilation techniques.

Acknowledgments. This research was supported by the National Ocean Partnership Program (NOPP) and the Office of Naval Research (ONR) under awards N00014-99-1-1048 and N00014-99-1-1066. The authors thank Adrian New for encouraging us to look at the modeled ventilation pathways and Jean Carpenter for her help in redrafting the figures.

\section{APPENDIX}

\section{Inclusion of Thermobaricity in Isopycnic-Coordinate Ocean Models}

A general functional relation between in situ specific volume and potential specific volume $\alpha_{r}(\theta, S)=\alpha(\theta$, $\left.S, p_{r}\right)$ is

$$
\alpha(\theta, S, p)=\alpha_{r}(\theta, S) \exp \int_{p}^{p_{r}} \kappa\left(\theta, S, p^{\prime}\right) d p^{\prime},
$$

where $\kappa$ is the compressibility:

$$
\kappa=-\frac{1}{\alpha}\left(\frac{\partial \alpha}{\partial p}\right)_{\theta, S}
$$

over the pressure interval $\left(p, p_{r}\right)$.

In order to isolate the components of density variability that are capable of creating circulation-inducing buoyancy contrasts from those that are dynamically passive, $\kappa$ is split into a pressure-dependent component $\kappa^{(p)}$ and a residual thermobaric component $\kappa^{(\theta)}$. The latter component has some salinity and pressure dependence as well.

Substituting $\kappa^{(p)}+\kappa^{(\theta)}$ for $\kappa$ in (A1) leads to

$$
\alpha(\theta, S, p)=\alpha_{r}(\theta, S) A_{r}(p) \exp \int_{p}^{p_{r}} \kappa^{(\theta)}\left(\theta, S, p^{\prime}\right) d p^{\prime},
$$

where

$$
A_{r}(p)=\exp \int_{p}^{p_{r}} \kappa^{(p)}\left(p^{\prime}\right) d p^{\prime}
$$

is the term whose effect we are trying to remove. We then define the "virtual potential specific volume" (the inverse of virtual potential density) as

$$
\alpha^{*}(\theta, S, p)=\frac{\alpha(\theta, S, p)}{A_{r}(p)} .
$$

The pressure gradient force in a hydrostatic fluid can be expressed as the isobaric gradient of the geopotential $\phi \equiv g z$, that is, $\alpha \boldsymbol{\nabla}_{z} p=\nabla_{p} \phi$. Integrating the hydrostatic equation $\partial \phi / \partial p=-\alpha$ from the sea surface to a pressure $p$ gives

$$
\begin{aligned}
-\alpha \boldsymbol{\nabla}_{z} p & =-\boldsymbol{\nabla}_{p} \phi \\
& =-\boldsymbol{\nabla} \phi_{\mathrm{srf}}+\int_{0}^{p} A_{r}\left(p^{\prime}\right) \boldsymbol{\nabla}_{p} \alpha^{*} d p^{\prime} .
\end{aligned}
$$

If a modified geopotential $\phi^{*}$ is defined by

$$
\frac{\partial \phi^{*}}{\partial p} \equiv-\alpha^{*},
$$

then

$$
-\boldsymbol{\nabla}_{p} \phi^{*}=-\boldsymbol{\nabla}_{p} \phi-\int_{0}^{p}\left[A_{r}\left(p^{\prime}\right)-1\right] \nabla_{p} \alpha^{*} d p^{\prime} .
$$

The factor $\left[A_{r}(p)-1\right]$ in a 5 -km-deep ocean is at most of order $10^{-2}$ when compared with the other terms and

$$
-\nabla_{p} \phi \simeq-\nabla_{p} \phi^{*} .
$$

This implies that estimates of the horizontal pressure gradient force are insensitive to whether the hydrostatic equation has been integrated using the in situ or the virtual potential density, that is, whether the oceanic density field is subject to compression by the pressurerelated part $\kappa^{(p)}$ of the compressibility coefficient. Hence, the frequency of buoyancy oscillations remains unchanged if the specific volume of seawater in the model is changed from its in situ value to $\alpha^{*}$. For details, the reader is referred to Sun et al. (1999).

\section{REFERENCES}

Blanke, B., and P. Delecluse, 1993: Variability of the tropical Atlantic Ocean simulated by a general circulation model with two different mixed-layer physics. J. Phys. Oceanogr., 23, 1363-1388.

Bleck, R., 2002: An oceanic general circulation model framed in hybrid isopycnic-Cartesian coordinates. Ocean Modell., 4, 5558.

_ - and D. B. Boudra, 1981: Initial testing of a numerical ocean circulation model using a hybrid (quasi-isopycnic) vertical coordinate. J. Phys. Oceanogr., 11, 755-770.

—_, and S. Benjamin, 1993: Regional weather prediction with a model combining terrain-following and isentropic coordinates. Part I: Model description. Mon. Wea. Rev., 121, 1770-1785.

—- H. P. Hanson, D. Hu, and E. B. Kraus, 1989: Mixed layer/ thermocline interaction in a three-dimensional isopycnal model. J. Phys. Oceanogr., 19, 1417-1439.

-, C. Rooth, D. Hu, and L. T. Smith, 1992: Salinity-driven transients in a wind- and thermohaline-forced isopycnic coordinate model of the North Atlantic. J. Phys. Oceanogr., 22, 1486-1505.

Böning, C. W., and P. Herrmann, 1994: Annual poleward heat transport in the ocean: Results from high-resolution modeling of the North and Equatorial Atlantic. J. Phys. Oceanogr., 24, 91-105.

- W. R. Holland, F. O. Bryan, G. Danabasoglu, and J. C. McWilliams, 1995: An overlooked problem in model simulations of the thermohaline circulation and heat transport in the Atlantic Ocean. J. Climate, 8, 515-523.

Bryan, F. O., and W. R. Holland, 1989: A high resolution simulation of the wind- and thermohaline-driven circulation in the North 
Atlantic Ocean. Parameterization of Small-Scale Processes: Proc. 'Aha Huliko'a Hawaiian Winter Workshop, Honolulu, HI, University of Hawaii at Manoa, 99-115.

Brydon, D., S. Sun, and R. Bleck, 1999: A new approximation of the equation of state for sea water, suitable for numerical ocean models. J. Geophys. Res., 104, 1537-1540.

Canuto, V. M., A. Howard, Y. Cheng, and M. S. Dubovikov, 2001: Ocean turbulence. Part I: One-point closure model-Momentum and heat vertical diffusivities. J. Phys. Oceanogr., 31, 14131426.

, ——, ——, and — 2002: Ocean turbulence. Part II: Vertical diffusivities of momentum, heat, salt, mass, and passive scalars. J. Phys. Oceanogr., 32, 240-264.

Chassignet, E. P., and Z. D. Garraffo, 2001: Viscosity parameterization and the Gulf Stream separation. From Stirring to Mixing in a Stratified Ocean: Proc. 'Aha Huliko'a Hawaiian Winter Workshop, Honolulu, HI, University of Hawaii at Manoa, 3741.

— , L. T. Smith, R. Bleck, and F. O. Bryan, 1996: A model comparison: Numerical simulations of the North and Equatorial Atlantic oceanic circulation in depth and isopycnic coordinates. $J$. Phys. Oceanogr., 26, 1849-1867.

— , and Coauthors, 2000: DAMÉE-NAB: The base experiments. Dyn. Atmos. Oceans, 32, 155-184.

Dietrich, G., K. Kalle, W. Krauss, and G. Siedler, 1980: General Oceanography, An Introduction. 2d ed. John Wiley, $626 \mathrm{pp}$.

Friedrich, H., and S. Levitus, 1972: An approximation to the equation of state for sea water, suitable for numerical ocean models. $J$. Phys. Oceanogr., 2, 514-517.

Garzoli, S. L., and E. J. Katz, 1983: The forced annual reversal of the Atlantic North Equatorial Countercurrent. J. Phys. Oceanogr., 13, 2082-2093.

Gent, P. R., and J. C. McWilliams, 1990: Isopycnal mixing in ocean circulation models. J. Phys. Oceanogr., 20, 150-155.

— J. Willebrand, T. J. McDougall, and J. C. McWilliams, 1995: Parameterizing eddy-induced tracer transports in ocean circulation models. J. Phys. Oceanogr., 25, 463-474.

Griffies, S. M., A. Gnanadesikan, R. C. Pacanowski, V. D. Larichev, J. K. Dukowicz, and R. D. Smith, 1998: Isoneutral diffusion in a z-coordinate ocean model. J. Phys. Oceanogr., 28, 805-830. , and Coauthors, 2000a: Developments in ocean climate modelling. Ocean Modell., 2, 123-192.

_ R. C. Pacanowksi, and R. W. Hallberg, 2000b: Spurious diapycnal mixing associated with advection in a $z$-coordinate ocean model. Mon. Wea. Rev., 128, 538-564.

Hall, M. M., and H. L. Bryden, 1982: Direct estimates and mechanisms of ocean heat transport. Deep-Sea Res., 29, 339-359.

Halliwell, G. R., 2003: Evaluation of vertical coordinate and vertical mixing algorithms in the HYbrid Coordinate Ocean Model (HYCOM). Ocean Modell., in press.

Han, Y.-J., 1984: A numerical world ocean general circulation model. Part II: A baroclinic experiment. Dyn. Atmos. Oceans, 8, 141172.

Hellerman, S., and M. Rosenstein, 1983: Normal monthly wind stress over the world ocean with error estimates. J. Phys. Oceanogr., 13, 1093-1104.

Holland, W. R., and F. O. Bryan, 1994: Sensitivity studies on the role of the ocean in climate change. Ocean Processes in Climate Dynamics: Global and Mediterranean Examples, P. MalanotteRizzoli and A. R. Robinson, Eds., Kluwer Academic, 111-134.

Jackett, D. R., and T. J. McDougall, 1995: Minimal adjustment of hydrographic profiles to achieve static stability. J. Atmos. Oceanic Technol., 12, 381-389.

Jia, Y., 2000: Formation of an Azores Current due to Mediterranean overflow in a modeling study of the North Atlantic. J. Phys. Oceanogr., 30, 2342-2358.

Kraus, E. B., and J. S. Turner, 1967: A one-dimensional model of the seasonal thermocline: II. The general theory and its consequences. Tellus, 19, 98-106.

Large, W. G., J. C. McWilliams, and S. C. Doney, 1994: Oceanic vertical mixing: A review and a model with a nonlocal boundary layer parameterization. Rev. Geophys., 32, 363-403.

—, G. Danabasoglu, S. C. Doney, and J. C. McWilliams, 1997: Sensitivity to surface forcing and boundary layer mixing in a global ocean model: Annual-mean climatology. J. Phys. Oceanogr., 27, 2418-2447.

Lazier, J. R. N., and D. G. Wright, 1993: Annual velocity variations in the Labrador Current. J. Phys. Oceanogr., 23, 659-678.

Levitus, S., 1982: Climatological Atlas of the World Ocean. NOAA Prof. Paper 13, 173 pp. and 17 microfiche.

MacDonald, A. M., and C. Wunsch, 1996: An estimate of global ocean circulation and heat fluxes. Nature, 382, 436-439.

Marsh, R., M. J. Roberts, R. A. Wood, and A. L. New, 1996: An intercomparison of a Bryan-Cox type ocean model and an isopycnic ocean model. Part II: The subtropical gyre and heat balances. J. Phys. Oceanogr., 26, 1528-1551.

McDougall, T. J., and P. C. McIntosh, 2001: The temporal-residualmean velocity. Part II: Isopycnal interpretation and the tracer and momentum equations. J. Phys. Oceanogr., 31, 1222-1246.

Mellor, G. L., and T. Yamada, 1982: Development of a turbulence closure model for geophysical fluid problems. Rev. Geophys. Space Phys., 20, 851-875.

New, A. L., Y. Jia, M. Coulibaly, and J. Dengg, 2001: On the role of the Azores Current in the ventilation of the North Atlantic Ocean. Progress in Oceanography, Vol. 48, Pergamon, 163-194.

Özgökmen, T. M., E. P. Chassignet, and C. G. H. Rooth, 2001: On the connection between the Mediterranean outflow and the Azores Current. J. Phys. Oceanogr., 31, 461-480.

Pickart, R. S., F. Straneo, and G. W. K. Moore, 2003: Is Labrador Sea Water formed in the Irminger Sea? Deep-Sea Res., 50, 23 52.

Price, J. F., R. A. Weller, and R. Pinkel, 1986: Diurnal cycling: Observations and models of the upper ocean response to diurnal heating, cooling, and wind mixing. J. Geophys. Res., 91, 84118427.

Roberts, M. J., R. Marsh, A. L. New, and R. A. Wood, 1996: An intercomparison of a Bryan-Cox type ocean model and an isopycnic ocean model. Part I: The subpolar gyre and high-latitude processes. J. Phys. Oceanogr., 26, 1495-1527.

Schmitz, W. J., Jr., 1996: On the world ocean circulation: Volume I. Woods Hole Oceanographic Institution Tech. Rep. WHOI-9603, Woods Hole, MA, $141 \mathrm{pp}$.

Schott, F. A., and C. W. Böning, 1991: The WOCE model in the western equatorial Atlantic: Upper layer circulation. J. Geophys. Res., 96, 6993-7004.

Smith, L. T., E. P. Chassignet, and R. Bleck, 2000a: The impact of lateral boundary conditions and horizontal resolution on North Atlantic water mass transformations and pathways in an isopycnic coordinate ocean model. J. Phys. Oceanogr., 30, 137159.

Smith, R. D., M. E. Maltrud, F. O. Bryan, and M. W. Hecht, 2000b: Numerical simulations of the North Atlantic Ocean at $1 / 10^{\circ} . J$. Phys. Oceanogr., 30, 1532-1561.

Sun, S., R. Bleck, C. G. H. Rooth, J. Dukowicz, E. P. Chassignet, and P. Killworth, 1999: Inclusion of thermobaricity in isopycniccoordinate ocean models. J. Phys. Oceanogr., 29, 2719-2729.

U.S. WOCE, 1993: U.S. WOCE implementation plan. U.S. WOCE Office Rep. 5, College Station, TX, 146 pp.

Willebrand, J., and Coauthors, 2001: Circulation characteristics in three eddy-permitting models of the North Atlantic. Progress in Oceanography, Vol. 48, Pergamon, 123-161. 